\title{
FORMATION AND EVOLUTION OF PLANETARY SYSTEMS: UPPER LIMITS TO THE GAS MASS IN DISKS AROUND SUN-LIKE STARS
}

\author{
I. PASCUCCI \\ Steward Observatory, University of Arizona, 933 North Cherry Avenue, Tucson, AZ 85721 \\ U. GORTI \\ Department of Astronomy, University of California, 601 Campbell Hall, Berkeley, CA 94720 \\ D. Hollenbach \\ NASA Ames Research Center, Moffett Field, CA 94035 \\ J. NAJITA \\ National Optical Astronomy Observatory, 950 North Cherry Avenue, Tucson, AZ 85719 \\ M. R. MEYER \\ Steward Observatory, University of Arizona, 933 North Cherry Avenue, Tucson, AZ 85721
}

J. M. Carpenter, L. A. Hillennbrand, G. J. Herczeg, and D. L. Padgett Astronomy Option, California Institute of Technology, MS 105-24, Pasadena, CA 91125

E. E. Mamajek

Harvard-Smithsonian Center for Astrophysics, 60 Garden Street, Cambridge, MA 02138

M. D. Silverstone, W. M. Schlingman, J. S. Kim, and E. B. Stobie Steward Observatory, University of Arizona, 933 North Cherry Avenue, Tucson, AZ 85721

J. Boumman, S. Wolf, And J. Rodmann

Max-Planck-Institut für Astronomie, Koenigstuhl 17, Heidelberg D-69117, Germany

D. C. Hines

Space Science Institute, 3100 Marine Street, Boulder, CO 80301

AND

J. Lunine and R. MaLhotra

Lunar and Planetary Laboratory, University of Arizona, 1629 East University Boulevard, Tucson, AZ 85721 Received 2006 April 10; accepted 2006 June 26

\begin{abstract}
We have carried out a sensitive search for gas emission lines at IR and millimeter wavelengths for a sample of 15 young Sun-like stars selected from our dust disk survey with Spitzer. We have used mid-IR lines to trace the warm $(300-100 \mathrm{~K})$ gas in the inner disk and millimeter transitions of ${ }^{12} \mathrm{CO}$ to probe the cold $(\sim 20 \mathrm{~K})$ outer disk. We report no gas line detections from our sample. Line flux upper limits are first converted to warm and cold gas mass limits using simple approximations allowing a direct comparison with values from the literature. We also present results from more sophisticated models following Gorti \& Hollenbach that confirm and extend our simple analysis. These models show that the $\left[\mathrm{S}_{\mathrm{I}}\right] 25.23 \mu \mathrm{m}$ line can set constraining limits on the gas surface density at the disk inner radius and traces disk regions up to a few AU. We find that none of the 15 systems have more than $0.04 M_{\mathrm{J}}$ of gas within a few AU from the disk inner radius for disk radii from 1 to $\sim 40 \mathrm{AU}$. These gas mass upper limits even in the eight systems younger than $\sim 30 \mathrm{Myr}$ suggest that most of the gas is dispersed early. The gas mass upper limits in the 10-40 AU region, which is mainly traced by our $\mathrm{CO}$ data, are $<2 M_{\oplus}$. If these systems are analogs of the solar system, they either have already formed Uranus- and Neptune-like planets or will not form them beyond 100 Myr. Finally, the gas surface density upper limits at $1 \mathrm{AU}$ are smaller than $0.01 \%$ of the minimum mass solar nebula for most of the sources. If terrestrial planets form frequently and their orbits are circularized by gas, then circularization occurs early.
\end{abstract}

Subject headings: circumstellar matter — infrared: stars — planetary systems: formation — solar system: formation

Online material: color figures

\section{INTRODUCTION}

Circumstellar disks are a natural outcome of the star formation process (Shu et al. 1987). Initially massive and gas dominated, they evolve into tenuous dusty disks possibly with embedded planets (e.g., Meyer et al. 2006). Studying the evolution of gas and dust in circumstellar disks is essential to understanding how planets form.
The properties of circumstellar dust have been extensively studied in young and old disks since the IRAS mission. The emerging evolutionary picture is of submicron interstellar grains that grow to larger sizes (up to kilometer-size bodies) and settle to the disk midplane (e.g., Bouwman et al. 2001; Przygodda et al. 2003; Apai et al. 2005). In a later stage, collisions between forming planets and/or minor bodies such as asteroids or Kuiper Belt 
objects can produce second-generation dust (or debris) in circumstellar disks (e.g., Rieke et al. 2005; Kim et al. 2005; Bryden et al. 2006). This evolution leads to tenuous dust disks where the emission from grains is optically thin to its own radiation. In contrast, less is known observationally about gas evolution in circumstellar disks.

The presence or absence of gas affects planet formation in profound ways. Gas masses between 1 and 10 times the dust masses in circumstellar disks control the dust dynamics and shape the disk structure (e.g., Takeuchi \& Artymowicz 2001). The gas lifetime constrains the time for forming gas giant planets like Jupiter and Saturn. In addition, the ultimate size and distribution of giant planets may be affected by the disk gas mass. Even during the assembly of terrestrial planets, which concluded approximately 30 Myr after the origin of the solar system (Jacobsen 2005), a few Earth masses of gas in the terrestrial planet region could have influenced the eccentricity and final size of growing planets (Kominami \& Ida 2004).

Observations of gas have mainly targeted young accreting disks (few Myr old) and probed the cold outer regions (outside $\sim 30$ AU) and the warm regions within $\sim 1$ AU of such disks. Cold gas $(20 \mathrm{~K}<T<50 \mathrm{~K})$ is traced in the millimeter by $\mathrm{CO}$ rotational lines that indirectly measure gas masses in young accreting disks with radii of few hundred AU (e.g., Dutrey \& Guilloteau 2004). The only bona fide debris disk detected in millimeter CO transitions is that around the A star 49 Cet (Zuckerman et al. 1995; Dent et al. 2005). Warm gas $(\sim 1000 \mathrm{~K})$ in the inner disk $(\leq 1 \mathrm{AU})$ is probed in the near-infrared by $\mathrm{CO}$ vibrational transitions (e.g., Najita et al. 2003). These transitions may also trace gas in the terrestrial planet zone of evolved tenuous disks. In addition, $\mathrm{H}_{2}$ rovibrational lines have been detected in a few disks and found to trace the surface layer of the disks at distances of 10-30 AU from the star (e.g., Bary et al. 2003). Observations in the ultraviolet have successfully detected a number of gas lines toward pre-main-sequence stars (e.g., Valenti et al. 2000, 2003). In particular, fluorescent $\mathrm{H}_{2}$ emission has been found tracing both warm gas $(\sim 2500 \mathrm{~K})$ at or near the disk surface within $\sim 1$ AU from the star (Herczeg et al. 2002) and surrounding molecular gas shocked in the interaction with stellar outflows (e.g., Walter et al. 2003). These UV diagnostics are not sensitive to the bulk of the much colder gas at larger radii. This cold gas can be traced with $\mathrm{H}_{2}$ absorption lines in the farultraviolet (FUV) but only in optically thin disks that are observed close to edge-on. Stringent upper limits to the line-of-sight $\mathrm{H}_{2}$ column density through the edge-on disks of $\beta$ Pic and AU Mic suggest that the primordial gas dispersed in less than $12 \mathrm{Myr}$ in both systems (Lecavelier des Etangs et al. 2001; Roberge et al. 2005).

One region not adequately probed by most of the observations described above is the $\sim 1-30$ AU region $(\sim 300-50 \mathrm{~K})$, corresponding to the giant planet-forming region in the solar system. The Infrared Space Observatory (ISO) provided a first glimpse. Thi et al. (2001b) reported pure rotational $\mathrm{H}_{2} S(0)$ and $S(1)$ line detections from a large number of pre-main-sequence stars and also from three main-sequence stars with debris disks. These detections translated into large reservoirs of gas, suggesting a gas dispersal timescale longer than the accretion timescale. However, subsequent ground-based infrared spectroscopy (Richter et al. 2002; Sheret et al. 2003; Sako et al. 2005) and UV observations (Lecavelier des Etangs et al. 2001) cast doubt on whether the observed lines originated in disks.

As part of the Formation and Evolution of Planetary Systems (FEPS) Spitzer legacy program, we are carrying out a compre- hensive survey of disks around Sun-like stars to characterize the gas dissipation timescale. In conjunction with this survey, Gorti \& Hollenbach (2004, hereafter GH04) constructed detailed gas and dust models of optically thin dust disks in order to compare observational results with model spectra. In Hollenbach et al. (2005, hereafter H05) we applied these models to FEPS observations of the disk around the $30 \mathrm{Myr}$ star HD 105 and showed that less than $1 M_{\mathrm{J}}$ of gas exists in the planet-forming region (between 1 and $40 \mathrm{AU}$ for a disk inner radius of $\sim 1 \mathrm{AU}$ ). In the following, we extend our analysis to 15 Sun-like stars surrounded by optically thin dust disks, most of which have ages in the range 5-100 Myr. The target selection and observational strategy are presented in $\S 2$. From the nondetection of atomic and molecular gas lines, we place stringent upper limits on the gas mass in the planet-forming zone ( $\S \S 3$ and 4$)$. We discuss the implications on the gas evolution timescale and on the formation of gas giant and terrestrial planets in $\S 5$. Our findings are summarized in $\S 6$.

\section{OBSERVATIONS AND DATA REDUCTION}

In this section we describe the target selection for our gas survey with Spitzer ( $\S 2.1)$ and the data reduction of the midinfrared spectra $(\S 2.2)$. In order to trace outer disk regions with gas colder than $50 \mathrm{~K}$, we complemented the Spitzer data of many sources with millimeter observations of ${ }^{12} \mathrm{CO}$ lines (§ 2.3).

\subsection{Target Selection and Observational Strategy}

We selected objects from the FEPS dusty disk survey of 328 stars based on two observational criteria and several ancillary parameters. We chose the nearest objects with ages mostly between 5 and $100 \mathrm{Myr}$ and those located in the lowest infrared backgrounds so that the observing time required to meet our goal would be minimized. Targets were chosen to span a range of infrared excess emission, X-ray luminosity, and spectral type (within the bounds of our program: $0.8-1.5 M_{\odot}$ ). We required that mid- to far-IR excess be detected in IRAS and ISO observations and extended the sample to excess sources discovered within FEPS. We also included additional young sources ( $\leq 30 \mathrm{Myr})$ lacking infrared excess emission. Here we focus on a subset of these sources, whose dusty disks are thin at optical wavelengths. Results on the optically thick dust disks will be reported in a future contribution. The properties of the optically thin sample are summarized in Table 1. Ten out of 15 sources have excess emission from circumstellar dust starting at wavelengths longer than $\sim 20 \mu \mathrm{m}$. Among the five sources with no excess emission, ScoPMS 214, AO Men, and V343 Nor were included in the sample because of their young ages $(\leq 15 \mathrm{Myr})$. Our MIPS and low-resolution IRS observations do not confirm the excess emission reported in the literature toward the older two sources HD 134319 and HD 216803 (Spangler et al. 2001; FajardoAcosta et al. 1999). In the case of HD 134319, the ISO $60 \mu \mathrm{m}$ flux was contaminated by a nearby source detected in our MIPS 24 and $70 \mu \mathrm{m}$ exposures.

Our Spitzer IRS observations were designed to be sensitive to less than $\sim 0.5 M_{\mathrm{J}}$ in molecular hydrogen gas at a temperature of $100 \mathrm{~K}$. Integration times were set to achieve a $5 \sigma$ detection against the noise. We used the IRS high-resolution modules providing $R \approx 700$ spectral resolution over 9.9-37.2 $\mu \mathrm{m}$. Note that the natural line width of the gas is expected to be $10-100$ times smaller than the $\sim 430 \mathrm{~km} \mathrm{~s}^{-1}$ resolution of the IRS highresolution spectra. The large-wavelength coverage of the IRS on Spitzer enables us to probe various atomic and molecular lines in addition to the molecular hydrogen transitions. In fact, GH04 show that certain lines, such as [S I] at $25.23 \mu \mathrm{m}$ and [S II] at $34.8 \mu \mathrm{m}$, 
TABLE 1

Summary of the Target Stellar Parameters

\begin{tabular}{|c|c|c|c|c|c|c|c|c|c|c|c|}
\hline Number & Source & $\begin{array}{c}\text { R.A. } \\
(\mathrm{J} 2000.0)\end{array}$ & $\begin{array}{c}\text { Decl. } \\
(\mathrm{J} 2000.0)\end{array}$ & Spectral Type & References & $\begin{array}{l}\text { Age } \\
\text { (Myr) }\end{array}$ & $\begin{array}{c}D \\
(\mathrm{pc})\end{array}$ & $\begin{array}{l}T_{\text {eff }} \\
(\mathrm{K})\end{array}$ & $\begin{array}{c}\log L_{\star} \\
\left(L_{\odot}\right)\end{array}$ & $\begin{array}{c}\log L_{\mathrm{X}} \\
\left(\text { ergs s }^{-1}\right)\end{array}$ & References \\
\hline 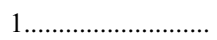 & ScoPMS $214^{\mathrm{a}}$ & 162949 & -215211.9 & K0 & 1 & $5^{\mathrm{b}}$ & 145 & 5318 & 0.26 & 30.72 & 11 \\
\hline З & MML 28 & 130151 & -530458.1 & $\mathrm{~K} 2$ & 2 & $17^{\mathrm{c}}$ & 108 & 4997 & -0.35 & 30.00 & 2 \\
\hline 4 & HD 37484 & 053740 & -283734.7 & F3 & 3 & 72 & 60 & 6727 & 0.55 & 29.07 & 12 \\
\hline 5................................. & HD 202917 & 212050 & $\begin{array}{lll}-53 & 02 & 03.1\end{array}$ & G5 & 4 & $30^{\mathrm{d}}$ & 46 & 5553 & -0.18 & 29.49 & 13 \\
\hline 6.1. & HD $134319^{\mathrm{a}}$ & 150550 & +640250.0 & G5 & 5 & 150 & 44 & 5716 & -0.14 & 29.23 & 11 \\
\hline 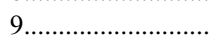 & HD 377 & 000826 & +063700.5 & G2 & 7 & 88 & 40 & 5852 & 0.09 & 29.10 & 11 \\
\hline 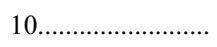 & $\mathrm{AO} \mathrm{Men}^{\mathrm{a}}$ & 061828 & -720241.6 & $\mathrm{~K} 3$ & 8 & $12^{\mathrm{e}}$ & 38 & 4359 & -0.59 & 30.16 & 14 \\
\hline 11 & HD 209253 & 220233 & -320801.6 & $\mathrm{~F} 6 / 7$ & 3 & 280 & 30 & 6217 & 0.21 & 29.70 & 15 \\
\hline 12 & HD 35850 & 052705 & -115403.4 & F $7 / 8$ & 9 & $12^{\mathrm{e}}$ & 27 & 6138 & 0.25 & 30.60 & 13 \\
\hline 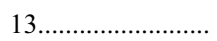 & HD 25457 & 040237 & -001608.2 & F7 & 10 & 110 & 19 & 6319 & 0.32 & 29.74 & 16 \\
\hline 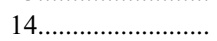 & HD 17925 & 025232 & -124611.2 & $\mathrm{~K} 1$ & 6 & 150 & 10 & 5173 & -0.43 & 28.97 & 17 \\
\hline
\end{tabular}

NoTEs.-Units of right ascension are hours, minutes, and seconds, and units of declination are degrees, arcminutes, and arcseconds. Targets are ordered by distance, from the farthest (ScoPMS 214) to the closest (HD 216803). Spectral types are from optical spectroscopy with accuracy of $0.5-1$ subtype (references in next column). The seventh column reports the stellar ages: for members of stellar groups we report the mean group ages (see notes below); otherwise, we report mean ages from L. A. Hillenbrand et al. (2006, in preparation). Typical errors are 30\% of the adopted age. All distances, except for ScoPMS 214, are from Hipparcos (Perryman et al. 1997); we assume the mean distance to Upper Sco (de Zeeuw et al. 1999) for ScoPMS 214. Effective temperatures are mainly from $B, V, K$ photometry, while bolometric luminosities are from the best-fit Kurucz stellar models to optical and near-infrared observations of the stellar photosphere (for the procedure we refer to J. M. Carpenter et al. 2006, in preparation). X-ray luminosities are reported in the eleventh column of the table, with references in the last column.

a These sources do not have excess emission at any of the observed wavelengths (see $\S 2.1$ for details).

b This source belongs to the Upper Scorpius group (Preibisch et al. 2002).

c These sources belong to the Lower Centaurus Crux association (Mamajek et al. 2002).

d These sources belong to the Tuc-Hor association (Zuckerman \& Webb 2000; Song et al. 2003; Zuckerman \& Song 2004; Moór et al. 2006).

e These sources belong to the $\beta$ Pic moving group (Zuckerman et al. 2001; Zuckerman \& Song 2004).

References.-(1) Walter et al. 1994; (2) Mamajek et al. 2002; (3) Houk 1982; (4) Houk \& Cowley 1975; (5) Vizier Online Data Catalog, III/206 (W. Buscombe 1998); (6) Houk \& Smith-Moore 1988; (7) Jaschek 1978; (8) Gray et al. 2006; (9) Houk \& Swift 1999; (10) Gray et al. 2003; (11) Voges et al. 1999; (12) Micela et al. 1997; (13) Wichmann et al. 2003; (14) Makarov 2003; (15) Tagliaferri et al. 1994; (16) Huensch et al. 1998; (17) Schmitt \& Liefke 2004.

are expected in many instances to be significantly stronger than the $\mathrm{H}_{2}$ lines.

\subsection{Data Reduction of the High-Resolution IRS Spectra}

Observations of our 15 targets were obtained between 2004 September and 2005 August. After our validation observations of HD 105 in 2003 December, in which we obtained only single on-source exposures, we switched to an on-source/off-source observing strategy to better subtract the background and improve source extraction. The objects have been all observed in the Fixed Cluster-Offsets mode with two nod positions on-source (located at $\frac{1}{3}$ and $\frac{2}{3}$ of the slit length) and two additional sky measurements acquired just after the on-source exposures. The IRS or PCRS peak-up options were used to place and hold the targets in the spectrograph slit with positional uncertainties always better than $1^{\prime \prime}$ ( $1 \sigma$ radial). We used the Short-High module (SH), covering the spectral range 9.9-19.6 $\mu \mathrm{m}$, and the LongHigh module (LH), covering the wavelength range between 18.7 and $37.2 \mu \mathrm{m}$. Both modules are cross-dispersed echelle spectrographs with spectral resolution of approximately 700 . A summary of the observational $\log$ is given in Table 2. The plate scale of the detector is 2.3 pixel $^{-1}$ for the SH module and 4 ".5 $\mathrm{pixel}^{-1}$ for the LH module. Emission at radii larger than $\sim 50 \mathrm{AU}$ could be spatially resolved with the SH module for targets closer than 20 pc (see Table 1). However, as shown in $\S 4.2$, mid-infrared lines only trace warm gas located within a few AU from the disk inner radius. Such emission is spatially unresolved for all targeted disks.

Raw high-resolution IRS data were processed with the Spitzer Science Center (SSC) pipeline S12.02. We start our data reduction from the droop products. Corrections applied in the SSC pipeline at this stage include saturation flagging, dark subtraction, linearity correction, cosmic-ray rejection, and integration ramp fitting. Further data reduction is based on self-developed IDL routines in combination with the SMART reduction package developed by the IRS Instrument Team at Cornell (Higdon et al. 2004). The first step consists of creating background-subtracted images from sky

TABLE 2

Log of the IRS High-Resolution Observations

\begin{tabular}{|c|c|c|c|}
\hline Number & AOR Key & $\begin{array}{c}\mathrm{SH} \\
R A M P \times n c y c l e s\end{array}$ & $\begin{array}{c}\text { LH } \\
R A M P \times n c y c l e s\end{array}$ \\
\hline ScoPMS $214 \ldots \ldots \ldots . .$. & 9776897 & $121.9 \times 6$ & $60.95 \times 8$ \\
\hline MML $17 \ldots \ldots \ldots \ldots$ & 13463296 & $121.9 \times 6$ & $60.95 \times 6$ \\
\hline MML $28 \ldots \ldots \ldots \ldots \ldots$ & 13462016 & $121.9 \times 6$ & $60.95 \times 7$ \\
\hline AO Men ...................... & 5458688 & $121.9 \times 2$ & $60.95 \times 2$ \\
\hline HD $35850 \ldots \ldots \ldots \ldots . . . . .$. & 9777920 & $31.46 \times 6$ & $14.68 \times 10$ \\
\hline 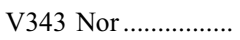 & 5458944 & $121.9 \times 2$ & $60.95 \times 2$ \\
\hline HD 12039 ................. & 13461760 & $121.9 \times 4$ & $60.95 \times 3$ \\
\hline HD 202917 ................. & 9778176 & $31.46 \times 5$ & $14.68 \times 8$ \\
\hline HD 25457 ................... & 9779712 & $6.29 \times 4$ & $6.29 \times 2$ \\
\hline HD 37484 .................... & 9780224 & $31.46 \times 10$ & $14.68 \times 10$ \\
\hline HD 377 .......................... & 13462272 & $121.9 \times 3$ & $60.95 \times 3$ \\
\hline HD $17925 \ldots \ldots \ldots \ldots \ldots . . . .$. & 9780480 & $31.46 \times 5$ & $14.68 \times 8$ \\
\hline HD $134319 \ldots \ldots \ldots \ldots . . . .$. & 9779968 & $31.46 \times 6$ & $14.68 \times 10$ \\
\hline HD $209253 \ldots \ldots \ldots \ldots . . . .$. & 9779200 & $31.46 \times 7$ & $14.68 \times 4$ \\
\hline HD $216803 \ldots \ldots \ldots \ldots . . .$. & 9777664 & $31.46 \times 5$ & $14.68 \times 8$ \\
\hline
\end{tabular}

NOTES.-The RAMP duration is the time in seconds per exposure. We acquired ncycles exposures before moving to the second slit position. The total onsource integration time is $R A M P \times n c y c l e s \times 2$. 
measurements acquired after the on-source exposures. ${ }^{1}$ Next we fix pixels marked bad in the "bmask" files with flag value equal to $2^{9}$ or larger, thus including anomalous pixels due to cosmic-ray saturation early in the integration, or preflagged as unresponsive. The SSC provides additional hot pixel masks for individual campaigns/modules that include permanently as well as temporarily hot pixels. We correct for these hot pixels using the IRSCLEAN package provided by the SSC. The routines recognize if a pixel is marked in both the bmasks and the additional hot pixel masks and correct only once. Bad and hot pixels are cleaned by averaging spatial profiles in rows above and below the affected row and then normalizing the average profile to that of the affected row. The profiles are fitted to the good data in the affected row by minimizing the $\chi^{2}$. These procedures have been extensively tested by the IRS GTO team and SSC. ${ }^{2}$ We use the background-subtracted pixel-corrected images to extract onedimensional spectra with the full aperture extraction routine in SMART.

We applied the same procedure to our sources and to calibrators observed within the Spitzer IRS Calibration program (PI: L. Armus). We used all of the calibrators processed with the S12.0.2 pipeline (two observations of HD 166780, three of HD 173511 , and one of $\xi$ Dra) to create two one-dimensional spectral response functions (one at each nod position) from the known stellar model atmosphere. The stellar models for the calibrators are available at the SSC Web site. ${ }^{3}$ The extracted spectra of each source are then divided by the spectral response function for each order and nod observation. The lower right corner of the chip is not well illuminated, resulting in a drop of signal at the end of each order. However, since the orders have a good overlap in wavelength, we simply trimmed the low-signal regions, retaining about $0.06 \mu \mathrm{m}$ overlap in the SH module and $0.1 \mu \mathrm{m}$ in the $\mathrm{LH}$ module. After trimming, spectra over all slit positions and cycles are averaged on an order basis. The uncertainties at each wavelength are estimated by the $1 \sigma$ standard deviations of the distribution of the data points used to calculate the mean spectrum over all cycles and nod positions and thus indicate the repeatability of our measurements. To these uncertainties we included the error on the derived spectral response function by taking the standard deviation of the calibrator spectra divided by the stellar atmospheres. The error quoted at overlapping wavelengths is the error propagation of the mean value. An example of a reduced spectrum is shown in Figure 1. All reduced spectra are available at the FEPS Web site. ${ }^{4}$ We report no detection of gas lines in any of these IRS spectra. We find that a few anomalous pixels remain in the reduced spectra that were not flagged in the bmasks or in the hot pixel masks. These pixels are typically located at the beginning and/or at the end of a spectral order and not at the location of the expected gas lines (see, e.g., the pixel at $27.78 \mu \mathrm{m}$ in Fig. 2).

\subsection{Millimeter CO Observations and Data Reduction}

Observations of ${ }^{12} \mathrm{CO}$ in $J=2-1(230.538 \mathrm{GHz})$ and $J=3-2$ $(345.796 \mathrm{GHz})$ were obtained with the $10 \mathrm{~m}$ Submillimeter Telescope (SMT) during two campaigns, 2003 December-2004 February and 2005 December-2006 January. The FWHM beam size of the SMT observations is $33^{\prime \prime}$ and $22^{\prime \prime}$ for the $J=2-1$ and $J=3-2$ transitions, respectively. The data were recorded using the CHIRP digital spectrometer (bandwidth of $\sim 215 \mathrm{MHz}$

\footnotetext{
${ }^{1}$ Because we have the same RAMP and number of cycles for the source and the sky, we subtracted a sky exposure from each on-source exposure.

2 See http://ssc.spitzer.caltech.edu/irs/roguepixels/.

3 See http://ssc.spitzer.caltech.edu/irs/calib/templ/.

4 See http://data.spitzer.caltech.edu/popular/feps/20051123_enhanced_v1/ir.
}

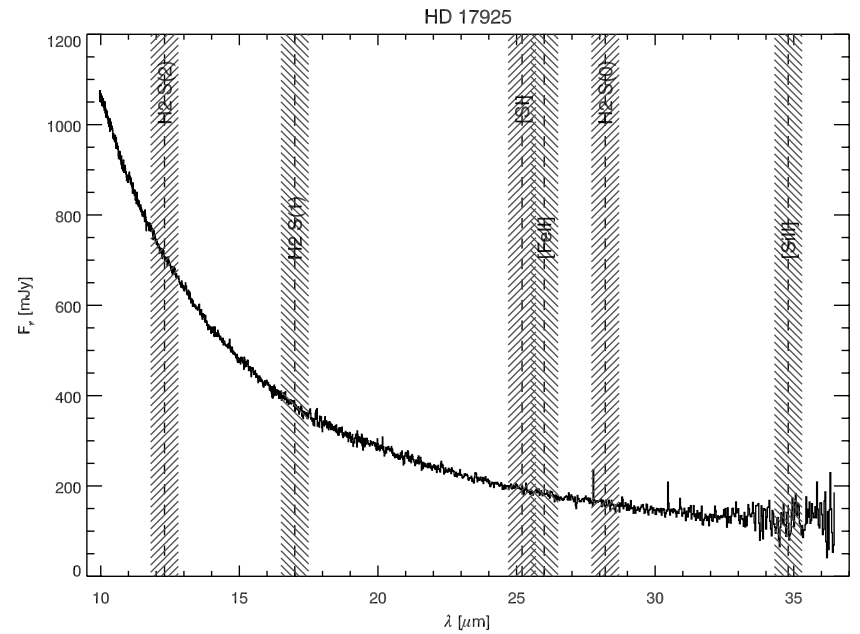

FIG. 1.- Spectrum of HD 17925 illustrating the location of the major molecular and atomic gas transitions (dashed lines) covered by the IRS high-resolution modules. The hatched area shows the region where we calculate the line flux upper limits (see $\S 3.1$ for more details). The continuum between 10 and $40 \mu \mathrm{m}$ is photospheric emission from HD 17925. Excess emission from dust was detected with $I S O$ at $60 \mu \mathrm{m}$ (Habing et al. 2001 and Fig. 4). The reduced spectra of all targets discussed here are available from the FEPS Web site (see footnote 4). [See the electronic edition of the Journal for a color version of this figure.]

and resolution of $\sim 40 \mathrm{kHz}$ ) and reduced using CLASS in the GILDAS $^{5}$ data reduction package. During data reduction, the spectra were smoothed to a velocity resolution of $0.2 \mathrm{~km} \mathrm{~s}^{-1}$, about 10 times smaller than the expected width of the line. A linear baseline was then removed from each spectrum, and all of the spectra for a given source were co-added by weighting each spectrum by $\mathrm{rms}^{-2}$. We calibrated our temperatures by measuring the ratio of observed and expected emission lines from planets (the latter was computed with the ASTRO software within GILDAS). These ratios, also known as beam efficiencies, had mean values of 0.77 and 0.50 for $J=2-1$ and $J=3-2$, respectively, during the first campaign and 0.74 and 0.55 for $J=2-1$ and $J=3-2$, respectively, during the second campaign. Repeated observations of spectral line calibrator sources during each campaign indicate that the dispersion in the calibration is $19 \%$ and $28 \%$ for $J=2-1$ and $J=3-2$, respectively. This dispersion was not included in the estimates of the line flux upper limits in $\S 3.2$. For two FEPS sources (HD 37484 and ScoPMS 214), the heliocentric velocity was unknown at the time of the observations, and the spectrometer was centered on $0.0 \mathrm{~km} \mathrm{~s}^{-1}$. The velocities were later determined from high-resolution optical spectra (White et al. 2006), and in all three instances, the velocities were well within the spectrometer bandwidth $\left(>100 \mathrm{~km} \mathrm{~s}^{-1}\right)$. We did not detect any $\mathrm{CO}$ emission line from any of our sources. The $1 \sigma$ upper limits per channel are provided in Table 3. Values are given in the main-beam scale; i.e., the antenna temperatures have been divided by the main-beam efficiencies of the telescope at the specific frequencies.

\section{SIMPLE ESTIMATES OF GAS MASS UPPER LIMITS}

We have not detected any lines associated with the gas in our IRS spectra, nor in our millimeter data. Line flux upper limits can be compared to model predictions of line strength and provide stringent constraints on the amount of gas in the planet-forming region. We pursue rigorous theoretical comparisons in $\S 4.2$.

\footnotetext{
5 See http://www.iram.fr/IRAMFR/GILDAS.
} 

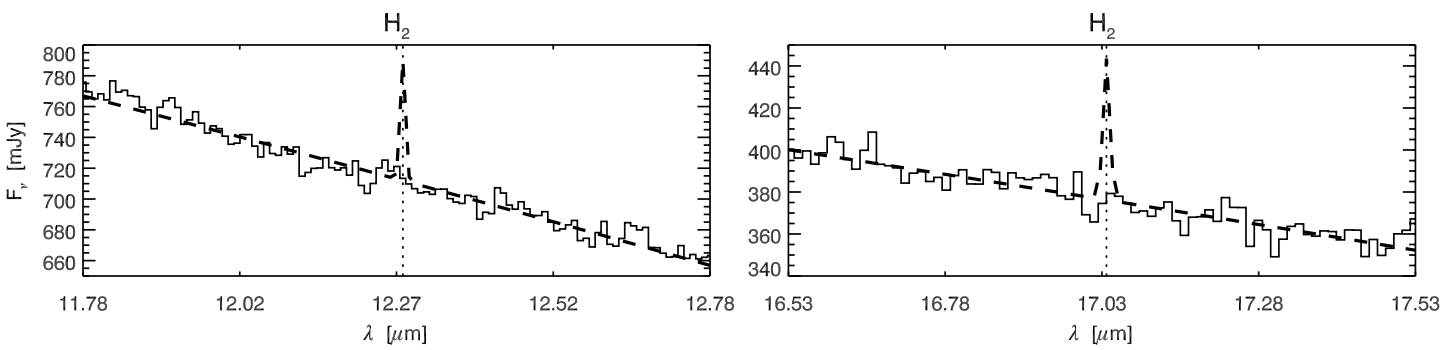

[SI]
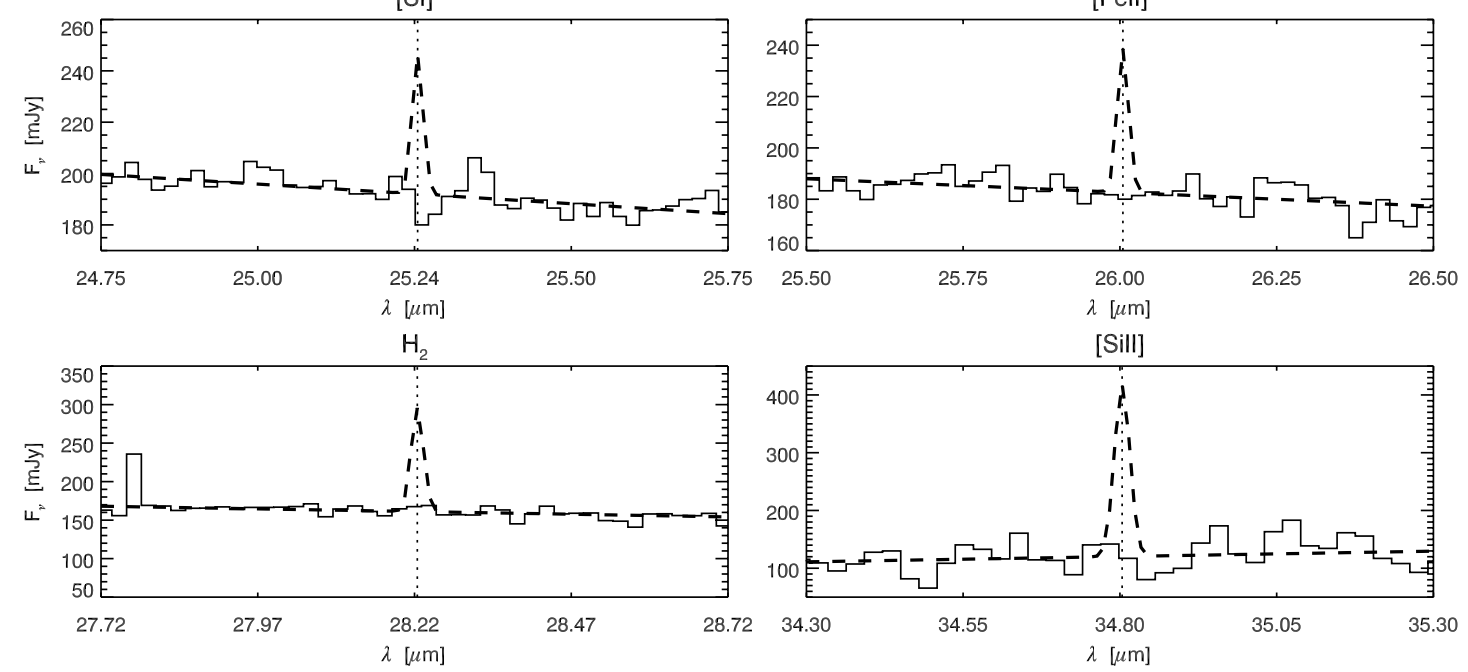

FIg. 2.-Expanded view of the wavelength regions around the expected lines toward the source HD 17925. Dashed lines are the hypothetical $5 \sigma$ line fluxes calculated assuming a resolution of 700 at all wavelengths. Lines are expected to be unresolved at all wavelengths. The pixel at $27.78 \mu \mathrm{m}$ is an example of an anomalous pixel that was not flagged in the bmasks or in the hot pixel masks.

Here for comparison to previous studies, we estimate gas mass upper limits in the approximation of optically thin emission at assumed fixed temperatures. Upper limits to the warm and cold gas are derived from the $\mathrm{H}_{2}(\S 3.1)$ and $\mathrm{CO}$ nondetections, respectively ( $\S 3.2)$. In addition, we calculate the upper limit on the radius of a putative gas disk under the assumption that the $\mathrm{CO}$ emission is optically thick in the inner regions $(\S 3.2)$. This radius will be later compared to that derived from our detailed modeling of gas disks that satisfy the observed infrared line flux limits.

TABLE 3

Millimeter Observations and Estimated $1 \sigma$ Upper Limits (PER Channel) IN K

\begin{tabular}{|c|c|c|c|}
\hline \multirow[b]{2}{*}{ Source } & \multirow{2}{*}{$\begin{array}{c}v_{\star}^{\mathrm{a}} \\
\left(\mathrm{km} \mathrm{s}^{-1}\right)\end{array}$} & \multicolumn{2}{|c|}{$\Delta T_{\mathrm{rms}}(\mathrm{K})$} \\
\hline & & $\mathrm{CO}(2-1)$ & $\mathrm{CO}(3-2)$ \\
\hline ScoPMS $214 \ldots \ldots \ldots \ldots . . . . .$. & -7.6 & $0.033^{\mathrm{b}}$ & 0.18 \\
\hline HD 35850 & 20.2 & 0.043 & $0.083^{\mathrm{b}}$ \\
\hline HD 12039 & 3.1 & 0.049 & 0.078 \\
\hline HD 25457 & 17.6 & $0.028^{\mathrm{b}}$ & 0.044 \\
\hline HD 37484 & 23.7 & $0.038^{\mathrm{b}}$ & \\
\hline HD 377 & -4 & 0.043 & $0.13^{\mathrm{b}}$ \\
\hline HD $17925 \ldots \ldots \ldots$ & 17.7 & $0.042^{b}$ & 0.056 \\
\hline HD 134319 & -6.8 & $0.06^{\mathrm{c}}$ & $0.068^{\mathrm{b}}$ \\
\hline HD $209253 \ldots \ldots \ldots$ & 8.0 & $\ldots$ & $0.095^{\mathrm{b}}$ \\
\hline
\end{tabular}

${ }^{a}$ The $v_{\star}$ are the radial heliocentric velocities of the stars as measured by stellar spectroscopy (White et al. 2006). Errors on $v_{\star}$ are about $0.5 \mathrm{~km} \mathrm{~s}^{-1}$ for all sources except for HD 377, where our uncertainty is $2 \mathrm{~km} \mathrm{~s}^{-1}$.

$\mathrm{b}$ Results from the first observational campaign (2003 December-2004 February).

${ }^{c}$ HD 134319 has been observed in the CO (2-1) transition by J. Najita \& J. P. Williams (2006, private communication) using the heterodyne receiver at JCMT (for the data reduction we refer to Najita \& Williams 2005).

\subsection{Warm Gas Limits from $\mathrm{H}_{2}$ Observations}

To estimate line flux upper limits from the mid-infrared observations, we first extract a $\pm 0.5 \mu \mathrm{m}$ region around the expected feature. Then we fit a baseline using a first-order polynomial. The $5 \sigma$ upper limits to the line flux are derived by taking the local rms dispersion of the pixels in the baseline-subtracted spectrum over 2 pixels per resolution element, assuming that the noise is uncorrelated. Note that because we calibrate our spectra from the average spectral response function of various standard stars, our upper limits partly include the error in the absolute flux calibration. We have tested that the line flux upper limits are not very sensitive to the width of the region where we fit the baseline. Choosing a region around the line that is $0.5 \mu \mathrm{m}$ smaller or larger than the $1 \mu \mathrm{m}$ width we adopt changes the upper limits on average by only $6 \% .{ }^{6}$ Our approach provides conservative upper limits. The use of a higher order polynomial to fit the continuum and/or the inclusion of the errors at each wavelength would lower the computed rms and thus the line flux upper limits. Our rms values are on average $20 \%$ higher than the error at the wavelengths of the expected lines. Table 4 summarizes the line flux upper limits for many of the transitions observed in the IRS modules: $\mathrm{H}_{2} S(2)$ at $12.28 \mu \mathrm{m}, \mathrm{H}_{2} S(1)$ at $17.04 \mu \mathrm{m}, \mathrm{H}_{2} S(0)$ at $28.22 \mu \mathrm{m},[\mathrm{S}$ I $]$ at $25.23 \mu \mathrm{m},[\mathrm{Fe}$ II] at $26.00 \mu \mathrm{m}$, and [Si II] at $34.80 \mu \mathrm{m}$. We show examples of hypothetical $5 \sigma$ lines in Figure 2 .

We convert $\mathrm{H}_{2}$ line flux upper limits into upper limits on the $\mathrm{H}_{2}$ mass (for the equations see, e.g., Thi et al. 2001a) assuming that the gas is in local thermal equilibrium, that the $\mathrm{H}_{2}$ lines are optically thin, and that the gas temperature is 100,150 , or $200 \mathrm{~K}$. Temperatures of $\approx 100 \mathrm{~K}$ correspond to the gas from which our

\footnotetext{
${ }^{6}$ These tests have been made on the three lines that are the most informative when compared to the gas models: the $\mathrm{H}_{2} S(1)$ at $17.04 \mu \mathrm{m}$, the $[\mathrm{S} \mathrm{I}]$ at $25.23 \mu \mathrm{m}$, and the $[\mathrm{Fe}$ II] at $26.00 \mu \mathrm{m}$.
} 
TABLE 4

Line Flux Upper Limits $(5 \sigma)$ from the High-Resolution IRS Spectra

\begin{tabular}{|c|c|c|c|c|c|c|}
\hline \multirow[b]{2}{*}{ Source } & \multicolumn{6}{|c|}{$\log \left[\right.$ line flux $\left.\left(\mathrm{W} \mathrm{cm}^{-2}\right)\right]$} \\
\hline & $\mathrm{H}_{2} S(2)$ & $\mathrm{H}_{2} S(1)$ & $\mathrm{H}_{2} S(0)$ & {$[\mathrm{S}$ I $]$} & {$[\mathrm{Fe} \mathrm{II}]$} & [Si II $]$ \\
\hline ScoPMS 214 & -21.73 & -21.89 & -21.41 & -21.57 & -21.57 & -20.99 \\
\hline 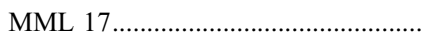 & -21.86 & -21.99 & -21.56 & -21.46 & -21.62 & -20.95 \\
\hline MML $28 \ldots . .$. & -21.95 & -22.02 & -21.51 & -21.60 & -20.27 & -20.81 \\
\hline HD 37484 & -21.13 & -21.28 & -21.20 & -21.18 & -21.36 & -20.76 \\
\hline 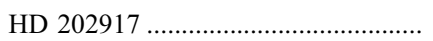 & -21.10 & -21.11 & -21.15 & -21.11 & -21.34 & -20.73 \\
\hline HD 134319 & -21.18 & -21.27 & -21.17 & -21.15 & -21.28 & -20.73 \\
\hline HD 12039 & -21.60 & -21.74 & -21.29 & -21.01 & -21.42 & -20.78 \\
\hline 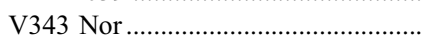 & -21.29 & -21.52 & -21.00 & -21.18 & -21.38 & -20.60 \\
\hline 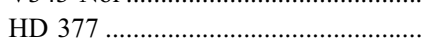 & -21.45 & -21.66 & -21.33 & -20.95 & -21.12 & -20.53 \\
\hline 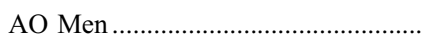 & -21.60 & -21.74 & -21.25 & -21.19 & -21.17 & -20.87 \\
\hline HD 209253 & -20.82 & -20.86 & -21.07 & -20.98 & -21.21 & -20.51 \\
\hline 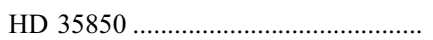 & -20.94 & -21.07 & -21.07 & -20.97 & -21.18 & -20.75 \\
\hline 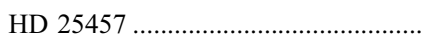 & -20.49 & -20.57 & -20.42 & -20.66 & -20.77 & -20.11 \\
\hline HD 17925 & -20.80 & -21.01 & -20.89 & -21.24 & -21.24 & -20.64 \\
\hline HD 216803 & -20.80 & -20.98 & -21.06 & -20.93 & -21.14 & -20.68 \\
\hline
\end{tabular}

own Jupiter and Saturn are thought to have formed (e.g., Gautier et al. 2001). Expanding the temperature range to $200 \mathrm{~K}$ allows us to make a direct comparison with published gas masses and upper limits for circumstellar disks (e.g., Thi et al. 2001a; Sako et al. 2005; Chen 2006; H05; Chen et al. 2006) and illustrate how gas masses depend on the assumed gas temperature. The $\mathrm{H}_{2} \mathrm{~S}(2)$ line does not set stringent limits for temperatures lower than $\sim 300 \mathrm{~K}$, the $\mathrm{H}_{2} S(1)$ transition is the most sensitive line at 150 and $200 \mathrm{~K}$, while the $\mathrm{H}_{2} S(0)$ and the $\mathrm{H}_{2} S(1)$ transitions provide similar values for gas at $100 \mathrm{~K}$. In Table 5 we report the lowest gas mass upper limits for temperatures of 100,150 , and $200 \mathrm{~K}$ and indicate whether they are derived from the $\mathrm{H}_{2} \mathrm{~S}(0)$ or the $\mathrm{H}_{2}$ $S(1)$ transitions for gas at $100 \mathrm{~K}$. This simple approach shows that gas masses are very sensitive to the assumed gas temperature: if the emitting gas is as cold as $100 \mathrm{~K}$, it takes about 100 times more mass to produce the measured line flux upper limits than if gas is at

TABLE 5

Gas Mass Upper Limits Estimated from the $\mathrm{H}_{2} S(0)$ and $\mathrm{H}_{2} S(1)$ Nondetections for Three Gas Temperatures

\begin{tabular}{|c|c|c|}
\hline Source & $\begin{array}{c}\mathrm{H}_{2} \text { Mass for } \\
100-150-200 \mathrm{~K} \text { Gas } \\
\left(M_{\oplus}\right)\end{array}$ & $\begin{array}{l}\text { Most Sensitive Line } \\
\text { for } 100 \mathrm{~K} \mathrm{Gas}^{\mathrm{a}}\end{array}$ \\
\hline ScoPMS $214 \ldots \ldots \ldots \ldots \ldots$ & $200-6.6-1.3$ & $\mathrm{H}_{2} S(1)$ \\
\hline MML 17................................. & $115-3.8-0.8$ & $\mathrm{H}_{2} S(1)$ \\
\hline MML 28................................... & $82-2.7-0.6$ & $\mathrm{H}_{2} S(1)$ \\
\hline HD 37484 & $64-4.6-0.9$ & $\mathrm{H}_{2} S(0)$ \\
\hline HD 202917 & $42-4.0-0.8$ & $\mathrm{H}_{2} \mathrm{~S}(0)$ \\
\hline HD 134319 & $37-2.5-0.5$ & $\mathrm{H}_{2} \mathrm{~S}(0)$ \\
\hline HD 12039 & $24-0.8-0.2$ & $\mathrm{H}_{2} S(1)$ \\
\hline 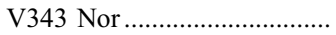 & $36-1.2-0.2$ & $\mathrm{H}_{2} \mathrm{~S}(1)$ \\
\hline HD 377 & $21-0.9-0.2$ & $\mathrm{H}_{2} S(0)$ \\
\hline AO Men & $19-0.6-0.1$ & $\mathrm{H}_{2} S(1)$ \\
\hline HD 209253 & $22-3.1-0.6$ & $\mathrm{H}_{2} S(0)$ \\
\hline HD 35850 & $17-1.5-0.3$ & $\mathrm{H}_{2} S(0)$ \\
\hline HD 25457 & $39-2.4-0.5$ & $\mathrm{H}_{2} S(1)$ \\
\hline HD 17925 & $4-0.2-0.05$ & $\mathrm{H}_{2} S(0)$ \\
\hline 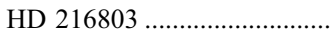 & $1-0.1-0.03$ & $\mathrm{H}_{2} S(0)$ \\
\hline
\end{tabular}

NOTE.-We used ortho-to-para ratios of 1.6, 2.5, and 3 for gas temperatures of 100,150 , and $200 \mathrm{~K}$, respectively (Sternberg \& Neufeld 1999).

${ }^{\text {a }}$ For gas temperatures of 150 and $200 \mathrm{~K}$ the $\mathrm{H}_{2} \mathrm{~S}(1)$ line is always more sensitive than the $\mathrm{H}_{2} \mathrm{~S}(0)$ and $\mathrm{H}_{2} S(2)$ lines.
$200 \mathrm{~K}$. Hence, it is very important to utilize detailed disk thermal models that describe the gas density and temperature distribution and that predict line fluxes from disks of a given mass for comparison with the observed upper limits on line fluxes.

Our measurements for optically thin disks are more sensitive than the ISO observations by Thi et al. (2001a) by at least a factor of 4 for sources at similar distance. The line detections claimed by Thi et al. (2001a) suggested that relatively large amounts $\left(6.7 M_{\mathrm{J}}-0.2 M_{\mathrm{J}}\right)$ of gas can persist into the debris-disk phase. Our gas mass upper limits show that even the six youngest disks in our sample (5-20 Myr) have less than 0.6 Jupiter masses of $100 \mathrm{~K}$ gas (or less than 0.005 Jupiter masses of $200 \mathrm{~K}$ gas). Our results are in agreement with the recent findings by Chen (2006), who reports masses of $100 \mathrm{~K}$ gas smaller than $15 M_{\oplus}\left(\right.$ or $\left.0.05 M_{\mathrm{J}}\right)$ in the disks of $\beta$ Pictoris ( $\sim 12$ Myr; Zuckerman et al. 2001) and 49 Ceti ( 10 Myr; Zuckerman et al. 1995). These low gas masses argue for short gas dispersal timescales.

Gas masses in Table 5 are provided mainly for comparison to previous studies. More detailed modeling of the dust and gas components is necessary to understand the mechanisms relevant to the gas heating, to determine gas temperatures, and thus to infer total gas masses from our nondetections. This procedure has been fully explored in H05 for HD 105, one of the first sources in the FEPS gas program, and is extended to our larger sample in $\S 4$.

\subsection{Cold Gas Limits from CO Observations}

For the millimeter observations, we convert the $1 \sigma$ noise into line flux upper limits as follows:

$$
\Delta F(\nu)=\frac{2 k \Delta T_{\mathrm{rms}}}{\lambda^{2}} \frac{\pi \theta^{2}}{4} d \nu \sqrt{N},
$$

where $k$ is the Boltzmann constant, $\Delta T_{\text {rms }}$ are our $1 \sigma$ upper limits per channel as reported in Table $3, \theta$ is the beam in radians at the transition $\lambda, d \nu$ is the frequency resolution $(d v=0.2 \mathrm{~km}$ $\mathrm{s}^{-1}$ ), and $N$ is the number of channels on which the line is distributed. Assuming a line width of $5 \mathrm{~km} \mathrm{~s}^{-1}$, which is the Keplerian velocity for gas at $\sim 30 \mathrm{AU}$ around a solar mass star, we have 25 channels for the SMT resolution of $0.2 \mathrm{~km} \mathrm{~s}^{-1}$. The $5 \sigma$ line flux upper limits are summarized in Table 6 and will be used here to provide an upper limit either to the $\mathrm{CO}$ mass in disks optically thin to the $\mathrm{CO}$ transitions or to the size of an optically thick $\mathrm{CO}$ 
TABLE 6

$5 \sigma$ Upper Limits for the Line Fluxes, CO Masses, and Outer Disk Radi

\begin{tabular}{|c|c|c|c|c|c|c|}
\hline \multirow[b]{2}{*}{ Source } & \multicolumn{2}{|c|}{$\log \left[\right.$ line flux $\left.\left(\mathrm{W} \mathrm{cm}^{-2}\right)\right]$} & \multicolumn{2}{|c|}{$M_{\mathrm{CO}}\left(M_{\oplus}\right)$} & \multicolumn{2}{|c|}{$R_{\text {out }}(\mathrm{AU})$} \\
\hline & $\mathrm{CO}(2-1)$ & $\mathrm{CO}(3-2)$ & $\mathrm{CO}(2-1)$ & $\mathrm{CO}(3-2)$ & $\mathrm{CO}(2-1)$ & $\mathrm{CO}(3-2)$ \\
\hline ScoPMS $214 \ldots \ldots \ldots \ldots \ldots$ & -23.43 & -22.51 & $1.2 \times 10^{-2}$ & $3.1 \times 10^{-2}$ & 236 & 403 \\
\hline 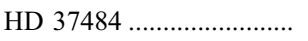 & -23.37 & $\ldots$ & $2.4 \times 10^{-3}$ & $\ldots$ & 105 & $\ldots$ \\
\hline 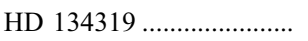 & -23.17 & -22.93 & $2.0 \times 10^{-3}$ & $1.1 \times 10^{-3}$ & 97 & 75 \\
\hline HD 12039 & -23.26 & -22.87 & $1.5 \times 10^{-3}$ & $1.1 \times 10^{-3}$ & 83 & 77 \\
\hline 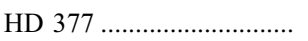 & -23.32 & -22.65 & $1.2 \times 10^{-3}$ & $1.7 \times 10^{-3}$ & 74 & 94 \\
\hline 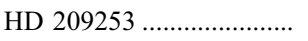 & $\ldots$ & -22.79 & $\ldots$ & $6.9 \times 10^{-4}$ & $\ldots$ & 61 \\
\hline HD 35850 & -23.32 & -22.85 & $5.4 \times 10^{-4}$ & $4.9 \times 10^{-4}$ & 50 & 51 \\
\hline 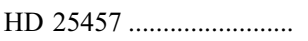 & -23.50 & -23.12 & $1.7 \times 10^{-4}$ & $1.3 \times 10^{-4}$ & 28 & 26 \\
\hline HD 17925 & -23.33 & -23.02 & $7.2 \times 10^{-5}$ & $4.5 \times 10^{-5}$ & 18 & 15 \\
\hline
\end{tabular}

Notes.- CO masses are calculated assuming optically thin emission. The outer disk radii are estimated from the assumption of $\mathrm{CO}$ being optically thick. For both estimates we adopted a gas temperature of $20 \mathrm{~K}$.

disk. We use our gas models to fully characterize the $\mathrm{CO}$ emission in $\S 4.2$.

Assuming that $\mathrm{CO}$ is optically thin throughout the disk, we can convert our line flux upper limits into CO mass upper limits. We use the formulation of Scoville et al. (1986) with appropriate changes to take into account different $\mathrm{CO}$ transitions and adopt an excitation temperature of $20 \mathrm{~K}$. We note that the $\mathrm{CO}$ mass is not very sensitive to the gas temperature and changes only by $\sim 20 \%$ if we assumed an excitation temperature of $40 \mathrm{~K}$. The $5 \sigma$ upper limits to the $\mathrm{CO}$ mass are summarized in Table 6. Our limiting values are typically a factor of 10 higher than those reported by Najita \& Williams (2005) for the disks around HD 104860 and HD 107146, due to a combination of shorter integration times and the assumption of larger line widths. Still these upper limits convert to less than a few $M_{\oplus}$ of total gas mass for many sources assuming the interstellar $\mathrm{H}_{2} / \mathrm{CO}$ number ratio of $10^{4}$. However, there are at least two important issues that need to be considered: the possible condensation of $\mathrm{CO}$ onto grains and the photodissociation of $\mathrm{CO}$ molecules. Both of these processes reduce the $\mathrm{CO}$ gas-phase abundance relative to $\mathrm{H}$ and thereby raise the upper limit on the total mass. Condensation of $\mathrm{CO}$ occurs for grain temperatures $\leq 50 \mathrm{~K}$ depending on the substrate onto which $\mathrm{CO}$ is adsorbed (for a discussion see, e.g., Najita \& Williams 2005). The lack of submillimeter continuum data for our sample does not allow us to constrain the temperature of the cold grains and thereby to estimate the effect of $\mathrm{CO}$ condensation. However, we show in $\S 4$ that photodissociation is a severe problem for tenuous gas disks such as those we have observed. If $\mathrm{CO}$ is optically thin but much of the gas-phase carbon is found in $\mathrm{C}^{+}$or $\mathrm{C}$, then the $\mathrm{CO}$ gas we detect would be representative of only a small fraction of the total gas mass, and the total gas mass associated with the optically thin $\mathrm{CO}$ could be $\gg$ few $M_{\oplus}$. In addition, the optically thin limits on $\mathrm{CO}$ mass tell us nothing about the $\mathrm{CO}$ mass located in the optically thick inner regions.

Assuming the existence of such an optically thick inner disk (which is indeed present in young accreting disks; Dutrey et al. 2004), we can set limits to the disk size by our CO flux upper limits. For simplicity we describe the $\mathrm{CO}$ emission as that from a blackbody times the solid angle subtended by a face-on $\operatorname{disk}^{7}$ and compare this emission to our $5 \sigma$ line flux upper limits. Table 6

\footnotetext{
${ }^{7}$ Because we do not have information on the disk inclinations, we assume face-on configurations here and in the following gas models. For optically thick lines the observed flux is proportional to the projected area of the disk. Thus, fluxes from optically thick lines can be corrected by multiplication with the cosine of the disk inclination.
}

summarizes the inferred upper limits on optically thick disk radii for a blackbody temperature of $20 \mathrm{~K}$. Disk radii are more sensitive to the assumed blackbody temperature than disk masses: a blackbody temperature of $40 \mathrm{~K}$ results in $\sim 35 \%$ smaller radii than those reported in Table 6. It is important to characterize the region inside which optically thick $\mathrm{CO}$ gas could exist because inside that region, gas masses could be high and still be undetected in CO. It is in these regions that the infrared upper limits may constrain the mass or surface density. In $\S 4$ we compare these radii, calculated for constant $T$, with those inferred by detailed gas models that compute the radial and vertical dependence of $T$ and the detailed radiative transfer operating for the $\mathrm{CO}$ transitions, as well as for the infrared transitions.

\section{CONSTRAINING THE GAS MASS IN THE PLANET-FORMING ZONE}

Even though $\mathrm{H}_{2}$ is the dominant constituent of the gas, $\mathrm{H}_{2}$ lines are not always the strongest transitions from circumstellar disks. Depending on the density, temperature, and chemical structure (which in turn depends on the radiation field and disk surface density distribution), other infrared atomic and molecular lines can have higher luminosities (GH04). In this section we make use of the line flux upper limits derived from the mid-infrared and millimeter $\mathrm{CO}$ transitions to derive constraining upper limits for the gas mass in the planet-forming zone.

H05 have shown that the dust disk of HD 105 is so tenuous that the dust does not affect the gas temperature or gas disk structure. In $\S 4.1$ we demonstrate that our systems also have too low dust surface density for gas-grain collisions to heat or cool the gas. Gas heating in these tenuous disks is dominated by X-rays and UV radiation from the central star; therefore, we can ignore the dust component in modeling the gas emission ( $\$ 4.2$ ). This is completely opposite to the case for young optically thick disks, where the gas temperature in much of the disk is coupled to the dust temperature by collisions, and where the surface gas is heated by the grain photoelectric heating mechanism (e.g., Jonkheid et al. 2004).

\subsection{Gas-Dust Collisions versus X-Ray Heating}

To understand whether dust may be an important heating mechanism for the gas, we need to evaluate the dust surface density of our disks. The observed infrared continuum excess from a disk gives an upper limit to the dust surface density for a chosen grain size. Ten of our gas sources have infrared excess emission at wavelengths longer than $\sim 20 \mu$ m with spectral energy distributions (SEDs) characteristic of optically thin dust disks (see Figs. 3 and 4). 


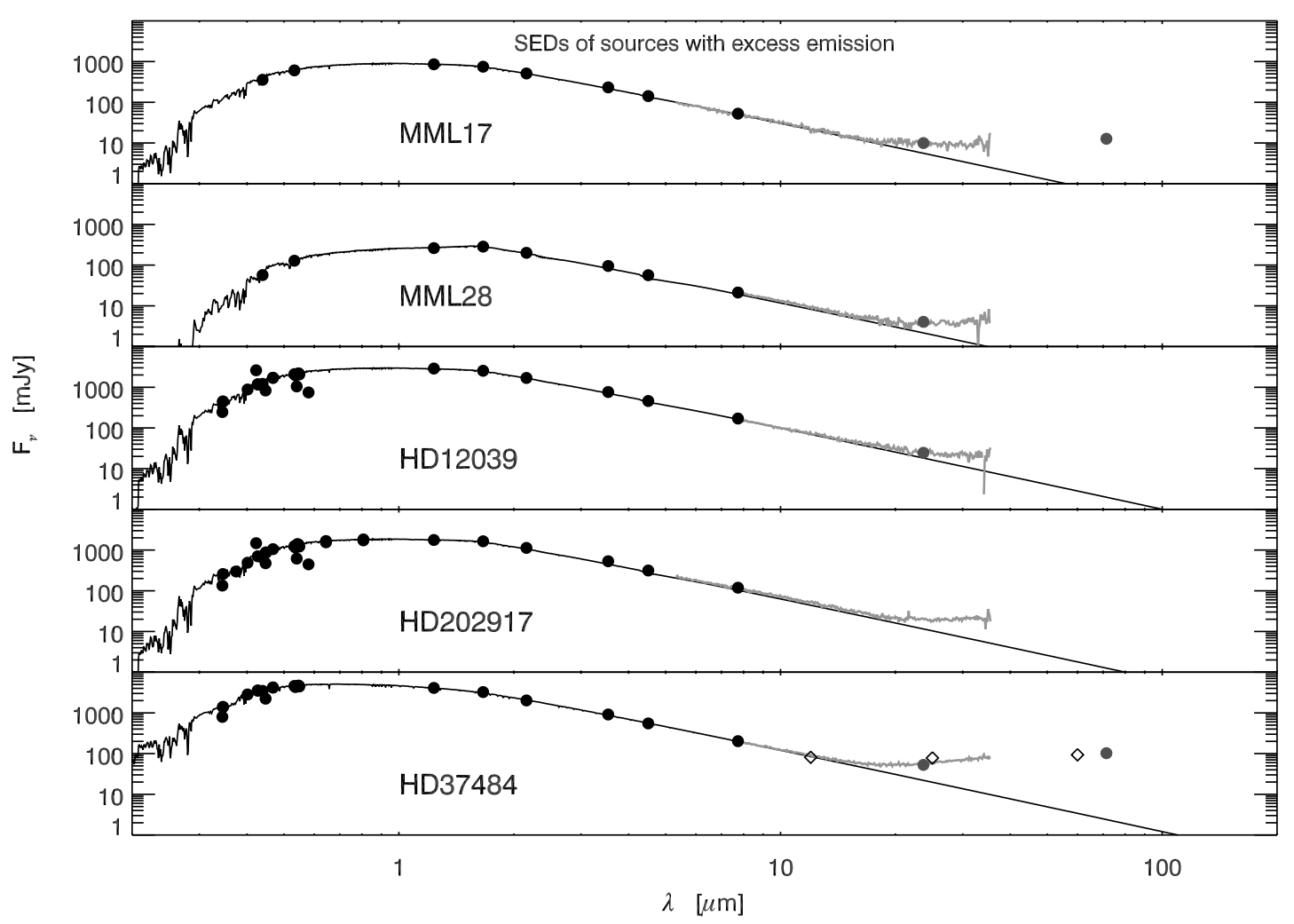

FIG. 3.- SEDs of sources with excess emission. Filled circles are IRAC, MIPS, and ground-based data points. Gray lines are IRS low-resolution spectra. IRAS and ISO

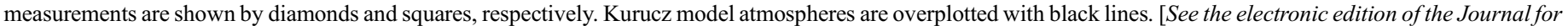
a color version of this figure.]

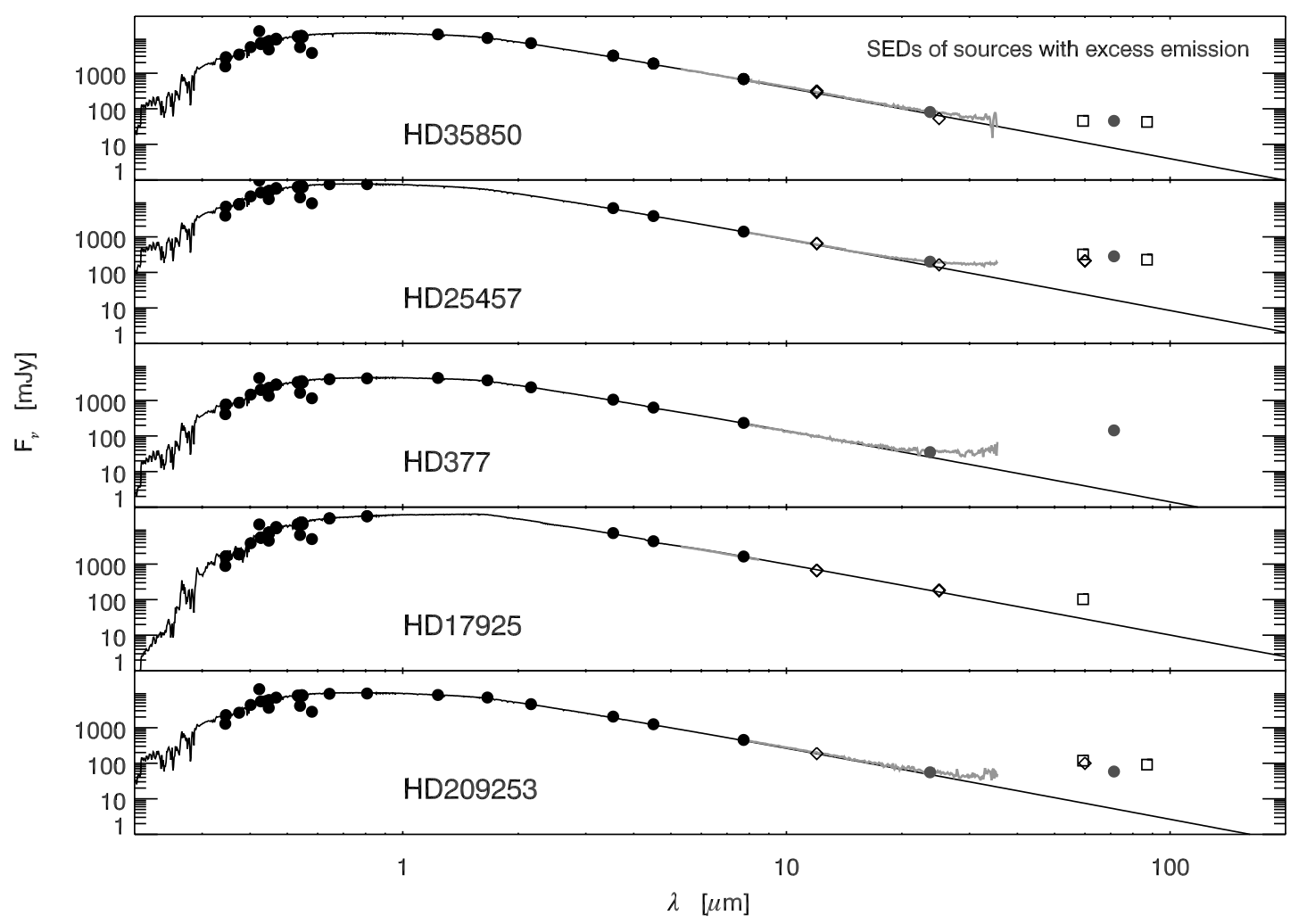

FIG. 4.- SEDs of sources with excess emission. See Fig. 3 for the plotting symbols. [See the electronic edition of the Journal for a color version of this figure.] 
We use HD 37484 and its disk as our demonstrative case because the system has the largest warm excess emission and the star has a low X-ray luminosity, thus representing the case with the largest dust surface density and the lowest X-ray heating of the gas. We assume that the disk has a dust surface density proportional to $r^{-1}$ (e.g., the Vega best-fit model to its debris disk by Su et al. 2005). We estimate in three steps the maximum dust surface density at the disk inner radius that is consistent with the infrared observations: (1) we calculate the dust temperature using the analytical solution of the radiative transfer equation for optically thin disks (e.g., Pascucci et al. 2004, eq. [5]); (2) we determine the excess emission by subtracting the best-fit Kurucz model atmosphere from the observed data; (3) we write the dust reemission in terms of the dust surface density at the inner radius (e.g., Wyatt et al. 1999, eq. [3]) and require that the reemission does not exceed the observed excess at any wavelength. The maximum dust surface density for inner radii from 1 to $19 \mathrm{AU}$ is shown in Figure 5 for a disk with small $(0.2 \mu \mathrm{m})$ and large $(2 \mu \mathrm{m})$ grains. The dust surface density for small grains is lower than that for large grains at all disk radii. This is because smaller grains are warmer than larger ones at the same distance from the star and because, for the same dust surface density, smaller grains have more total surface area than larger grains.

On the other hand, a lower limit to the dust surface density is obtained if the dust is to dominate the heating by X-rays. Heating from gas-dust collisions is proportional to the product of the densities of dust and gas, while X-ray heating is only proportional to the density of gas. By equating the contribution from gas-dust collisional heating and X-ray heating (see GH04 for the analytic forms of these heating processes), we obtain a minimum dust density for dust-gas collisions to contribute as much as X-ray heating. We compare the two heating mechanisms at the midplane of the disk where the number density is highest at any given radius, and hence where collisional heating is maximum. This is also the region where X-ray heating is at its lowest, since the attenuation is highest. To convert the number density to surface density, we use the mass of spherical Draine \& Lee (1984) silicate grains and a disk scale height of 0.1 times the radial distance, which is a good approximation for the inner regions of flared disks (e.g., Chiang \& Goldreich 1997). The resulting minimum dust surface densities for different inner disk radii and for two grain sizes are summarized in Figure 5. These values are obtained for a gas temperature of $70 \mathrm{~K}$. Note that the general trend will not change if a different gas temperature is assumed, but for gas hotter than the dust at any radius gas-dust collisions will result in cooling rather than heating the gas. However, the magnitude of the collisional cooling is similar to the magnitude of the collisional heating, the energy transfer being proportional to $T_{\text {gas }}-T_{\text {dust }}$.

The comparison of the two surface densities proves that our dust disks are too tenuous for dust to appreciably affect gas heating or cooling. This simple approach shows that at least 2 orders of magnitude higher dust surface densities are necessary for dust-gas collisions to equal X-ray heating. Because the other gas sources have lower excess emission than HD 37484 and/or higher X-ray luminosities, this result extends to the entire sample. We also point out that grain photoelectric heating is completely negligible for optically thin dust disks where most of the dust grains have grown to sizes larger than the very small $(\ll 0.2 \mu \mathrm{m})$ interstellar grains that dominate grain photoelectric heating (see the case of HD 105 by H05 and Fig. 3 of GH04). We therefore proceed to model the gas temperature and density structure of disks, ignoring their dust properties.

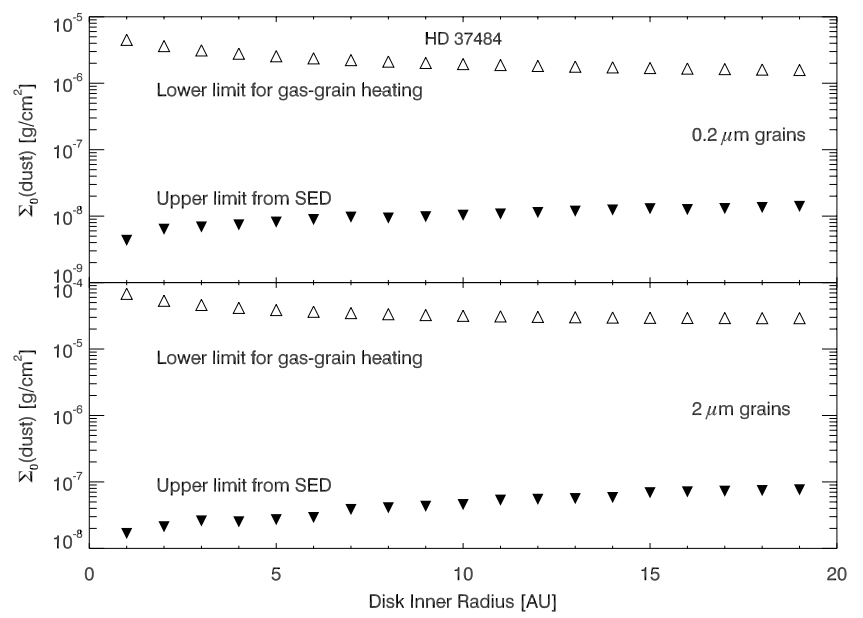

FIG. 5.-Comparison of dust surface densities at different disk inner radii for the source HD 37484. Open triangles indicate the minimum surface density for dust to contribute as much as X-rays in heating the gas at $70 \mathrm{~K}$. Downwardpointing filled triangles are the surface density upper limits imposed by the observed excess emission. For the dust, we adopted silicate grains with two sizes $(0.2$ and $2 \mu \mathrm{m})$ and opacities from Draine \& Lee (1984). Note that there are at least 2 orders of magnitude between the limits inferred from the SED modeling and from gas-grain heating at any disk inner radius. This shows that gas-grain collisions are an insignificant heating source in these tenuous dust disks. They also cannot dominate the cooling of the X-ray-heated gas (see text).

\subsection{Gas Models for Optically Thin Disks}

The simple approximations adopted in $\S \S 3.1$ and 3.2 allowed us to obtain initial estimates of gas mass upper limits and compare them to values published in the literature. The upper limits were shown to depend strongly on the assumed gas temperature. We can improve on these estimates by applying comprehensive gas models to calculate the gas disk temperature and thus infer total gas mass upper limits for the targeted disks. We use the models of GH04 that include the chemistry and thermal balance in a self-consistent manner and calculate the vertical density structure and the temperature in the radial and vertical directions. These models include heating and cooling from gas-dust collisions, stellar and interstellar UV radiation, stellar X-rays, collisional de-excitation of vibrationally excited $\mathrm{H}_{2}$ molecules, grain photoelectric heating, exothermic chemical processes, and cosmic rays. Condensation of molecules onto grains is not implemented for two main reasons. First, infrared lines originate from regions where dust is too hot $(\geq 100 \mathrm{~K})$ for condensation to occur appreciably. Second, the code is steady state but would require time-dependent solutions to properly treat the condensation of molecules. Gas-phase species are assumed to have interstellar abundances (Savage \& Sembach 1996 and Appendix A of GH04). We demonstrated in $\S 4.1$ that gas-dust collisions and grain photoelectric heating are insignificant heating and cooling mechanisms in tenuous dust disks; therefore, we removed the dust component in the models that follow.

Fiducial disk model.-We first consider a fiducial face-on gas disk extending from 1 to $100 \mathrm{AU}$ with a surface density dependence of $\Sigma \propto r^{-1}$, as indicated from observations of young disks (e.g., Dutrey et al. 1996). The main input parameters to the gas models are the stellar properties reported in Table 1 and stellar FUV fluxes (UV fluxes between $6 \mathrm{eV}<h \nu<13.6 \mathrm{eV}$ ). Our first modeling of the disk around HD 105 showed that stellar X-ray and UV photons dominate the gas heating in tenuous dust disks (the UV heats not by the grain photoelectric heating mechanism but by pumping $\mathrm{H}_{2}$ and by photodissociating and photoionizing 
TABLE 7

Summary of UV Luminosities and Templates

\begin{tabular}{|c|c|c|c|c|c|}
\hline \multicolumn{2}{|c|}{ FEPS SOURCES } & \multicolumn{3}{|c|}{ UV Templates } & \multirow[b]{2}{*}{ REFERENCE } \\
\hline Source & $\log \left(L_{\mathrm{UV}} / L_{\star}\right)$ & Source & Spectral Type & Criteria & \\
\hline 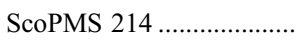 & -2.2 & ScoPMS $52^{\mathrm{a}}$ & K0 IV & Age, colors & 1 \\
\hline MML $17 \ldots \ldots \ldots \ldots \ldots \ldots \ldots$ & -2.4 & HD $146516^{\mathrm{a}}$ & G0 IV & Age, colors & 1 \\
\hline 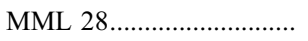 & -2.6 & ScoPMS $52^{\mathrm{a}}$ & K0 IV & Age, colors & 1 \\
\hline 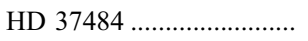 & -2.4 & HD 28568 & $\mathrm{~F} 2 \mathrm{~V}$ & X-ray & 2 \\
\hline 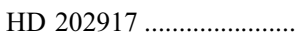 & -2.6 & HD 43162 & G5 V & X-ray & 2 \\
\hline HD 134319 & -3.2 & HD 134319 & $\ldots$ & $\ldots$ & 3 \\
\hline 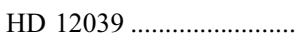 & -2.8 & HD 43162 & G5 V & X-ray & 2 \\
\hline 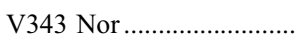 & -3.3 & V343 Nor & $\ldots$ & $\ldots$ & 3 \\
\hline 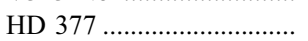 & -3.7 & EK Dra & $\mathrm{G} 1.5 \mathrm{~V}$ & Age, X-ray & 4 \\
\hline 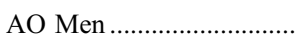 & -2.5 & RE J0137+18A & $\mathrm{K} 3 \mathrm{~V}$ & Age, colors & 3 \\
\hline HD 209253 & -2.8 & HD 33262 & F7 V & X-ray & 2 \\
\hline 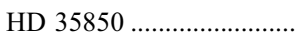 & -2.8 & HD 35850 & $\ldots$ & $\ldots$ & 3 \\
\hline 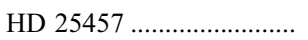 & -3.1 & HD 28033 & F8 V & X-ray & 2 \\
\hline 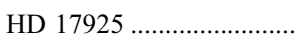 & -3.5 & HD 17925 & $\ldots$ & $\ldots$ & 3 \\
\hline 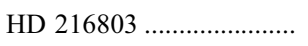 & -2.2 & HD 216803 & $\ldots$ & $\ldots$ & 3 \\
\hline
\end{tabular}

Nоте.-The UV luminosity we report here is calculated between 6.0 and $13.6 \mathrm{eV}$ for comparison to the interstellar radiation field (Habing 1969).

${ }^{a}$ These two template stars belong to the Upper Scorpius association $(d=145 \mathrm{pc})$ and have large visual extinctions as estimated from fitting their photometric data with Kurucz stellar atmospheres $\left(A_{V}=1.5\right.$ for ScoPMS 52 and $A_{V}=0.8$ for HD 146516). Their IUE spectra have been dereddened using the reddening law by Mathis (1990) before scaling them to the Kurucz model atmospheres of our targets. All other template stars have distances less than $65 \mathrm{pc}$ and thus negligible extinction: their IUE spectra scaled to the source distances and radii were found to match well the source Kurucz model atmospheres at wavelengths longward of $3200 \AA$. 2005

ReFERENCES.-(1) Valenti et al. 2000; (2) Wood et al. 2005; (3) IUE archive at http://archive.stsci.edu/iue/; (4) Ribas et al.

molecules and atoms; H05). Because stellar X-ray and UV fluxes depend on the stellar activity, we tried to collect measurements for each source. X-ray data are available for all targets (see Table 1), while only five of our sources have been observed in the UV by the International Ultraviolet Explorer (IUE). For these sources we merged the IUE data with the Kurucz model atmospheres ${ }^{8}$ and estimated the flux in eight energy bins (from 0.7 to $13.6 \mathrm{eV}$ ) that are relevant for the photodissociation and photoionization of dominant atomic and molecular species like $\mathrm{S}, \mathrm{Fe}, \mathrm{Mg}, \mathrm{Si}, \mathrm{CO}$, and $\mathrm{H}_{2}$. Where we lack UV data for our targets, we searched for stars with similar spectral type, age, and colors in the IUE archive and used them as templates for the FUV flux. For sources where ages are not well determined we used the spectral type and X-ray luminosity as the main criteria for assigning templates. We estimated the $\mathrm{H}$ I Ly $\alpha$ flux from the X-ray flux, based on the empirical relationship by Wood et al. (2005). Since Ly $\alpha$ emission contributes most of the FUV emission from late-type stars, we use it as a proxy for the total FUV emission and scale the templates accordingly, yielding order-of-magnitude estimates for the stellar UV field. A summary of UV templates and luminosities is given in Table 7. In $\S 4.3$ we discuss the dependence of our results on the input parameters that are most uncertain: the disk inner radius, the surface density slope, and the stellar UV luminosity.

Method.-We calculate the line fluxes from gas models with the total disk mass as our main variable parameter. For each transition reported in Tables 4 and 6, we find a disk mass where the calculated line flux from the models matches the observed flux limit. We determine the radius, $R_{\mathrm{em}}$, from within which $90 \%$ of the emission originates for each transition and also define a disk mass associated with this region, $M_{\mathrm{em}}$, which includes mass from the inner radius to $R_{\mathrm{em}}$. We also calculate the radius $R_{\text {thick }}$

\footnotetext{
${ }^{8}$ The Kurucz model atmospheres for the stellar parameters in Table 1 match in all cases the long-wavelength $I U E$ data (from $\sim 3000 \AA$ ). We used the $I U E$ data for wavelengths $<3000 \AA$ and the Kurucz model atmospheres for longer wavelengths.
}

within which the $\mathrm{CO}$ emission is optically thick. This region corresponds to a vertical column density of $\mathrm{CO}$ molecules of $10^{15}-10^{16} \mathrm{~cm}^{-2}$ (this value is similar for the two CO lines and corresponds to line optical depths larger than 1). Our modeling results are summarized in Tables 8 and 9 .

Results from the mid-infrared lines.-We find that the [S I] transition at $25.23 \mu \mathrm{m}$ sets the most stringent upper limits on the gas mass in comparison to the limits set by the other mid-infrared lines. We explored the sensitivity of our results to the S abundance in the gas phase using the disk of HD 35850 as a test case. Observations from comet Halley dust suggest that about half of the S could be in FeS grains (e.g., Pollack et al. 1994). Some S could be removed from the gas-phase component of circumstellar disks in this way, as recently proposed by Keller et al. (2002). However, current observations cannot quantify the amount of S into grains. To investigate a pessimistic case, we reduced the $\mathrm{S}$ abundance in gas phase by $90 \%$. This reduction results in a 5 times larger gas mass upper limit. This is because for such a low-mass disk the $\left[\mathrm{S}_{\mathrm{I}}\right]$ line is nearly optically thin (optical depths of $2-3$ ), so that the [S I] flux drops nearly proportional to the gas mass. Note that even if [S I] upper limits would be increased by a factor of 5, they would still be more stringent than those inferred from $\mathrm{H}_{2}$ lines for most of the sources (see below).

Our gas models predict also relatively strong [ $\mathrm{Si}$ II] lines at $34.8 \mu \mathrm{m}$; however, the IRS spectra become noisier at longer wavelengths and our line flux upper limits are not as low. These results are in agreement with what we found for the disk around HD $105(\mathrm{H} 05)$. The [ $\mathrm{Fe}$ II] line at $26 \mu \mathrm{m}$ is the second most sensitive transition and can set useful gas mass upper limits for two-thirds of the sample (in other words, in one-third of the sample, our models did not produce detectable [ $\mathrm{Fe}$ II] no matter how much gas mass was contained within the disk). The $\mathrm{H}_{2} \mathrm{~S}(1)$ line at $17 \mu \mathrm{m}$ provides the most stringent upper limits when compared to the other two $\mathrm{H}_{2}$ lines; nevertheless, gas masses can be constrained only for one-third of the sample with this diagnostic. 
TABLE 8

Results Derived from the Spitzer Observations and the Gas Models

\begin{tabular}{|c|c|c|c|c|c|c|c|c|c|c|}
\hline \multirow[b]{2}{*}{ SOURCE } & \multicolumn{3}{|c|}{$\Sigma_{0}\left(\mathrm{~g} \mathrm{~cm}^{-2}\right)$} & \multicolumn{3}{|c|}{$R_{\mathrm{em}}(\mathrm{AU})$} & \multicolumn{3}{|c|}{$M_{\mathrm{em}}\left(M_{\oplus}\right)$} & \multirow{2}{*}{$\begin{array}{l}M_{\text {disk }} \\
\left(M_{\oplus}\right)\end{array}$} \\
\hline & $\mathrm{H}_{2}$ & {$[\mathrm{Fe} \mathrm{II}]$} & {$\left[\begin{array}{ll}\mathrm{S} & \mathrm{I}\end{array}\right]$} & $\mathrm{H}_{2}$ & {$[\mathrm{Fe}$ II] } & {$[\mathrm{S}$ I $]$} & $\mathrm{H}_{2}$ & {$[\mathrm{Fe}$ II $]$} & {$\left[\begin{array}{ll}\mathrm{S} & \mathrm{I}\end{array}\right]$} & \\
\hline 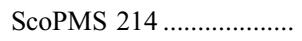 & $\ldots$ & $\ldots$ & 0.41 & $\ldots$ & $\ldots$ & 3.4 & $\ldots$ & $\ldots$ & 0.23 & 9.6 \\
\hline 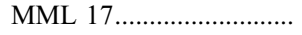 & $\ldots$ & $\ldots$ & 0.29 & $\ldots$ & $\ldots$ & 2.9 & $\ldots$ & $\ldots$ & 0.13 & 6.8 \\
\hline 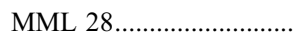 & $\ldots$ & $\ldots$ & 0.13 & $\ldots$ & $\ldots$ & 2.4 & $\ldots$ & $\ldots$ & 0.04 & 3.0 \\
\hline 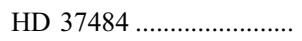 & $\ldots$ & 0.68 & 0.13 & $\ldots$ & 2.3 & 1.6 & $\ldots$ & 0.21 & 0.02 & 3.0 \\
\hline HD $202917 \ldots \ldots \ldots \ldots \ldots . .$. & $\ldots$ & 0.48 & 0.26 & $\ldots$ & 2.0 & 2.9 & $\ldots$ & 0.11 & 0.12 & 6.1 \\
\hline 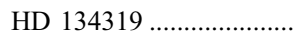 & $\ldots$ & $\ldots$ & 0.13 & $\ldots$ & $\ldots$ & 1.7 & $\ldots$ & & 0.02 & 3.0 \\
\hline 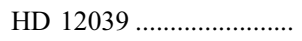 & 0.7 & 0.12 & 0.25 & 2.8 & 3.4 & 2.6 & 0.3 & 0.07 & 0.09 & 5.8 \\
\hline 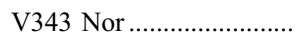 & $\ldots$ & 0.05 & 0.09 & $\ldots$ & 4.6 & 5.1 & $\ldots$ & 0.04 & 0.09 & 2.1 \\
\hline 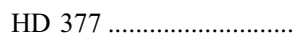 & 1.3 & $\ldots$ & 0.12 & 1.4 & $\ldots$ & 1.6 & 0.1 & $\ldots$ & 0.02 & 2.8 \\
\hline 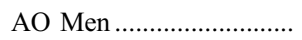 & 0.9 & 0.13 & 0.09 & 4.2 & 2.7 & 3.3 & 0.7 & 0.05 & 0.05 & 2.1 \\
\hline 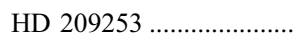 & $\ldots$ & 0.29 & 0.06 & $\ldots$ & 1.8 & 2.5 & $\ldots$ & 0.06 & 0.02 & 1.4 \\
\hline 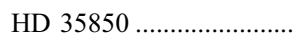 & 1.2 & 0.04 & 0.11 & 2.5 & 4.2 & 5.5 & 0.4 & 0.03 & 0.12 & 2.6 \\
\hline 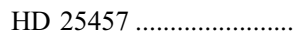 & $\ldots$ & 0.41 & 0.07 & $\ldots$ & 1.7 & 2.9 & $\ldots$ & 0.07 & 0.03 & 1.6 \\
\hline 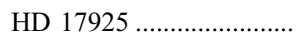 & $\ldots$ & 0.36 & 0.02 & $\ldots$ & 1.4 & 1.8 & $\ldots$ & 0.03 & 0.004 & 0.5 \\
\hline 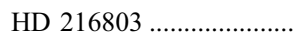 & 0.3 & 0.07 & 0.08 & 2.5 & 1.5 & 1.9 & 0.1 & 0.008 & 0.02 & 1.9 \\
\hline
\end{tabular}

Notes.-Upper limits to the gas obtained from the line flux upper limits of $\mathrm{H}_{2}$ at $17 \mu \mathrm{m}$, [Fe II] at $26 \mu \mathrm{m}$, and [S I] at $25.23 \mu \mathrm{m}$ and our fiducial disk model. $\Sigma_{0}$ is the gas surface density at the disk inner radius ( $\left.1 \mathrm{AU}\right) . R_{\mathrm{em}}$ is the radius within which $90 \%$ of the emission originates. $M_{\mathrm{em}}$ is the gas mass between $1 \mathrm{AU}$ and $R_{\mathrm{em}}$. Total disk masses from the [S I] upper limits (last column) and our fiducial disk range from $0.5 M_{\oplus}$ (for HD 17925) to $8 M_{\oplus}$ (for ScoPMS 214). However, note that they are not tightly constrained by the Spitzer observations alone, which are only sensitive to gas between the inner disk radius and $R_{\mathrm{em}}$.

This is because the transition probabilities of the $\mathrm{H}_{2}$ lines are orders of magnitude smaller than those of the $\left[\mathrm{S}_{\mathrm{I}}\right]$ and $[\mathrm{Fe} \mathrm{II}$ transitions, while their excitation temperatures are quite similar. The $\mathrm{H}_{2}$ surface density upper limits are typically a factor of 10 higher than the surface densities set by [S I], while the [Fe II] transitions provide values similar to those from $\left[\mathrm{S}_{\mathrm{I}}\right]$ for many sources.

For an inner disk radius of $1 \mathrm{AU}$, the models indicate that the emitting region for the mid-infrared transitions is typically a few $\mathrm{AU}$ and extends up to $\sim 5 \mathrm{AU}$ in a few of our fiducial disks, suggesting that we are tracing a region analogous to that between Earth and Jupiter in our solar system. We note that the gas mass upper limits inferred from modeling the $\left[\mathrm{S}_{\mathrm{I}}\right]$ lines are less than $0.4 M_{\oplus}$ in the 1-5 AU region for all sources and are as low as $1 / 10$ of an Earth mass for two-thirds of the sample.

Comparing gas masses from the models to the simple LTE approximation $(\S 3.1)$ requires knowing the characteristic tem- perature of the emitting gas. For the five sources where we could set limits from the $\mathrm{H}_{2} S(1)$ line, we computed mean temperatures as follows:

$$
\langle T\rangle=\frac{\sum F_{i} T_{i}}{\sum F_{i}},
$$

where the sum is carried out over the spatial grid and the flux is the $\mathrm{H}_{2} \mathrm{~S}(1)$ flux. We obtain mean temperatures between $230 \mathrm{~K}$ (for HD 35850) and $160 \mathrm{~K}$ (for HD 216803). This temperature range reflects the different source heating; K-type stars like HD 17925 should have even lower $\langle T\rangle$ close to $100 \mathrm{~K}$. Limits on the mass of the emitting gas from modeling the [S I] line $\left(M_{\mathrm{em}}\right.$ in Table 8 ) are typically a factor of 50 and 10 lower than the warm gas limits calculated in $\S 3.1$ for gas temperatures of 150 and $200 \mathrm{~K}$, respectively. The difference results from the use of $\left[\mathrm{S} \mathrm{I}_{\mathrm{I}}\right]$ as the

TABLE 9

Results Derived from the SMT Observations and the Gas Models

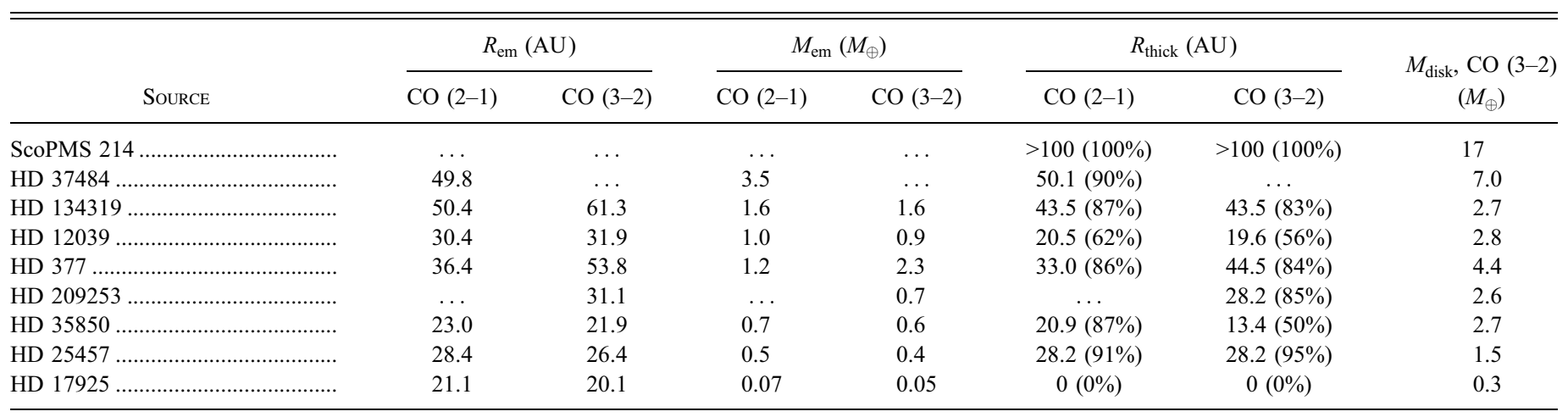

NoтеS.-Upper limits to the gas obtained from the SMT observations. $R_{\mathrm{em}}$ is the radius within which $90 \%$ of the emission originates. $M_{\mathrm{em}}$ is the mass between $1 \mathrm{AU}$ and $R_{\mathrm{em}}$. The CO (2-1) and CO (3-2) transitions trace colder gas in comparison to mid-infrared lines and thus are sensitive to the outer disk regions. $R_{\mathrm{thick}}$ is the radius at which the $\mathrm{CO}$ line optical depth is equal to 1 , corresponding to a column density of $\mathrm{CO}$ molecules (perpendicular to the disk, along the vertical direction) approximately equal to $10^{15} \mathrm{~cm}^{-2}$. The number in parentheses gives the percentage of optically thick emission in comparison to the total CO emission. The last column gives the total disk (hydrogenic) masses from 1 to $100 \mathrm{AU}$ for our fiducial disk surface density. These values are computed from the CO (3-2) transitions for all sources except ScoPMS 214 and HD 37484, where we used CO (2-1) data. In the case of ScoPMS 214 the CO (3-2) line flux upper limits are not stringent enough to set useful gas mass upper limits. For this gas mass the $\mathrm{CO}$ emission is optically thick all the way out to $100 \mathrm{AU}$. In the case of HD 37484 we have only CO (2-1) data. 


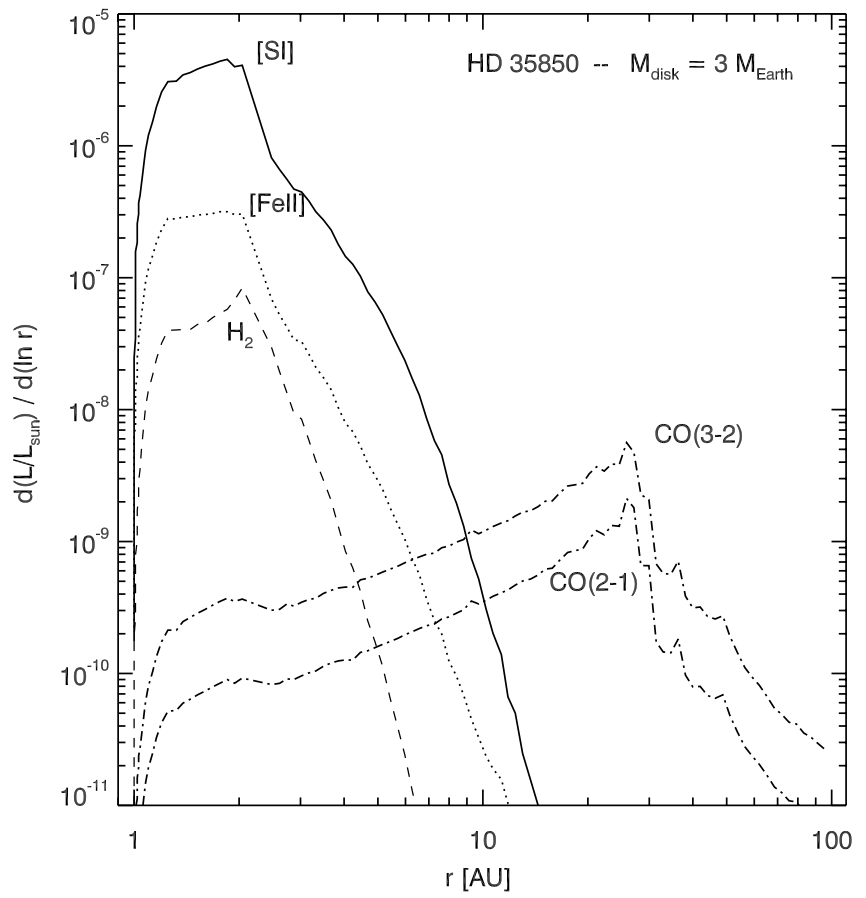

FIG. 6.-Emitting regions for mid-infrared and millimeter transitions. Plotted is the increment in line luminosity within a logarithmic radial annulus vs. the disk radius. The luminosities of the $\mathrm{H}_{2}$ (dashed line), [Fe II] (dotted line), and [S I] (solid line) transitions steeply increase at the disk inner radius, remain high out to a few AU, and rapidly decline at larger radii. The luminosities of millimeter CO transitions (dot-dashed lines) increase more gradually with radius. The drop in luminosity at $\sim 30$ AU occurs because CO becomes optically thin to its own radiation at these radii and is rapidly photodissociated. The wiggles are due to the coarse grid spacing used for the calculation in the outer regions.

tracer and the temperature structure in real disks. The limits on the total gas mass in a disk depend on the power law of the gas surface density and on the outer disk radius. For our fiducial disk model $\left(\Sigma \propto r^{-1}, 1 \mathrm{AU} \leq r \leq 100 \mathrm{AU}\right)$ upper limits to the total gas mass range from $10 \bar{M}_{\oplus}$ (for ScoPMS 214) to $0.5 M_{\oplus}$ (for HD 17925), with higher limits typically for the more distant sources, which are also the younger (see last column of Table 8).

Results from the millimeter $\mathrm{CO}$ lines.-While mid-infrared line luminosities trace the inner few AU of circumstellar disks, millimeter CO transitions are sensitive to colder gas located in the outer disks. CO is mainly heated by stellar X-ray and UV emission, but at large disk radii $\left(\sim 20 \mathrm{AU}\right.$ for a star with $L_{\mathrm{UV}} \sim$ $10^{-3} L_{\odot}$ ) the interstellar UV field can dominate the local stellar UV. Figure 6 shows the line luminosity per logarithmic radial annulus produced in a disk as a function of $r$. Mid-infrared line luminosities increase steeply at the disk inner radius, peak in the inner few AU, and rapidly fall as the temperature decreases. In contrast, CO line luminosities gradually increase with radius, peak at larger disk radii, and decline as $\mathrm{CO}$ begins to photodissociate. CO emission is so widespread that radii as large as $20 \mathrm{AU}$ and up to $60 \mathrm{AU}$ (see $R_{\mathrm{em}}$ of Table 9) are required to encompass $90 \%$ of the CO luminosity. This emission is mostly optically thick, suggesting that as a first approximation $\mathrm{CO}$ is mainly tracing the radius inside of which the $\mathrm{CO}$ is optically thick rather than the total disk mass. The disk radii estimated with the simple assumption of optically thick $\mathrm{CO}$ emission and $20 \mathrm{~K}$ temperature are typically a factor of 2 higher (and can be up to a factor of 4 higher; e.g., HD $12039)$ than the $R_{\text {thick }}$ from the models. This suggests that gas temperatures as high as $\sim 50 \mathrm{~K}$ are more representative of the region where $\mathrm{CO}$ is optically thick (see also the discussion in $\S 3.2$ ). In the case of HD 17925 the low observed flux limits only

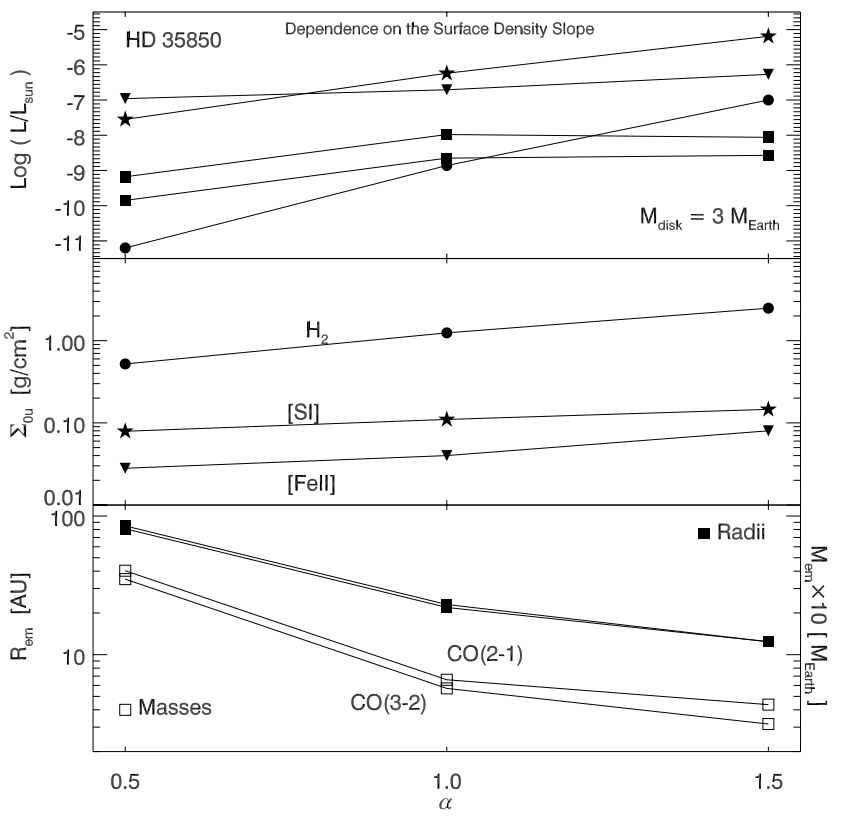

FIG. 7.-Dependence of our results on the surface density power law $\left(\Sigma \propto r^{-\alpha}\right)$. Changes in line luminosities for a disk of $3 M_{\oplus}$ around HD 35850 are plotted in the top panel. Stars, triangles, circles, and squares denote lines luminosities for the [S I], [ Fe II], $\mathrm{H}_{2} S(1)$, and $\mathrm{CO}$ transitions, respectively. The middle panel shows the variation in the surface density upper limits at $1 \mathrm{AU}\left(\Sigma_{0 u}\right)$. Steeper density slopes $(\alpha=1.5)$ increase the mid-infrared line luminosities and lower the total gas mass upper limits. However, the surface density at the inner radius also increases with $\alpha$ for a given disk mass, which is why the limits on the surface density are higher for $\alpha=1.5$ (see also text). The extension (filled squares) and mass (open squares) of the region emitting in the millimeter are plotted in the bottom panel.

allow a very tenuous gas disk. The low column density of the disk results in photodissociation of CO by the interstellar UV field (the average $\mathrm{H}_{2} / \mathrm{CO}$ number ratio is $10^{5}$, an order of magnitude higher than the interstellar value), and $\mathrm{CO}$ emission from this disk is optically thin at all radii.

\subsection{Dependence of the Results on Uncertain Input Parameters}

In this section we test the dependence of our results on the slope of the gas surface density, on the stellar UV field, and on the disk inner radius, which are the main input parameters to our gas models lacking independent observational constraints.

First, we consider different slopes of the gas surface density power law, $\Sigma(r) \propto r^{-\alpha}$, and use the disk around HD 35850 as the demonstrative case. In addition to our fiducial disk model with $\alpha=1$, we summarize in Figure 7 results for a flatter $(\alpha=0.5)$ and a steeper ( $\alpha=1.5$ ) surface density distribution, thus comprising the observed range of surface density slopes in circumstellar disks. For a disk with fixed mass, distributing more mass in the inner regions $(\alpha=1.5)$ results in increased mid-infrared line luminosities (Fig. 7, top panel). But a steeper density slope reduces the radius $R_{\mathrm{em}}$ of the emitting region and results in higher surface density upper limits at $1 \mathrm{AU}$ (Fig. 7, middle panel). For instance, the $R_{\mathrm{em}}$ for the [S I] line decreases from 10.4 AU for $\alpha=0.5$ to $3.7 \mathrm{AU}$ for $\alpha=1.5$, with the intermediate value of 5.5 AU for our fiducial disk (see Table 8). The $\mathrm{H}_{2} \mathrm{~S}(1)$ transition is more sensitive to the redistribution of mass than the [Fe II] and [S I] lines because it is the least optically thick transition. Differences between the limiting surface densities are less than a factor of 2 for $\alpha$ differing by 0.5 (Fig. 7, middle panel). CO lines are not affected appreciably for $\alpha=1$ or larger, but distributing more mass in the outer regions $(\alpha=0.5)$ results in a more extended CO 


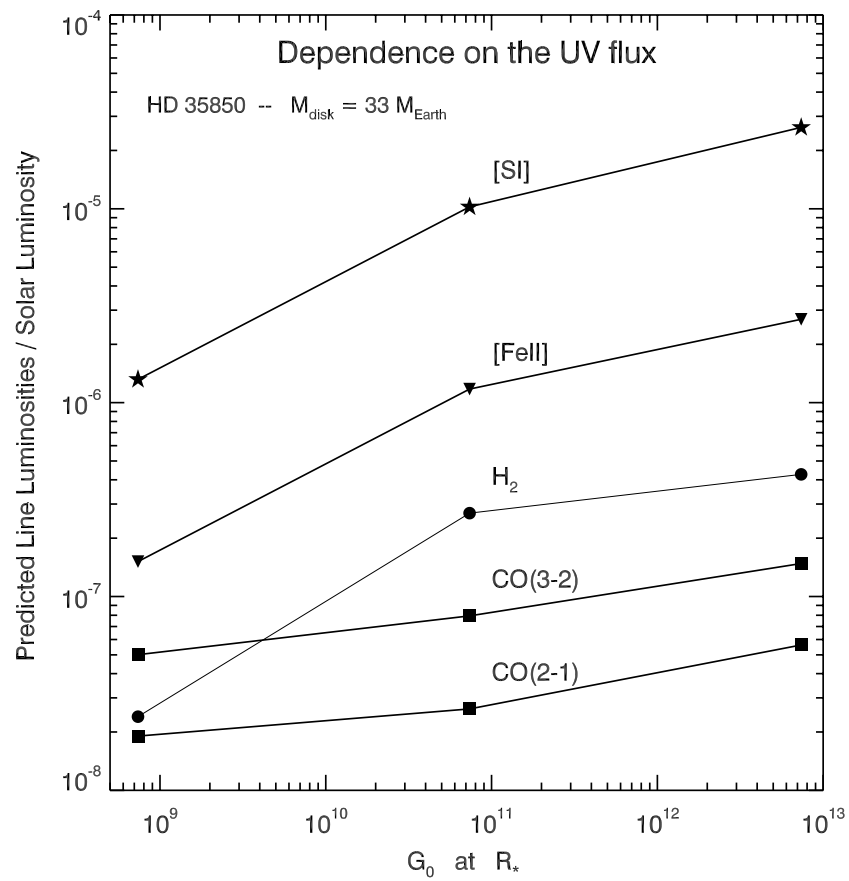

FIG. 8.-Dependence of line luminosities on the UV flux (see Fig. 7 for the symbols). These luminosities have been computed for a disk of $33 M_{\oplus}$ (from 1 to $100 \mathrm{AU}$ ) around the star HD 35850. $G_{0}$ is the UV flux between 6 and $13.6 \mathrm{eV}$ at the stellar surface normalized to the interstellar "Habing flux" $\left(1.6 \times 10^{-3} \mathrm{ergs}\right.$ $\left.\mathrm{cm}^{-2} \mathrm{~s}^{-1}\right)$.

emission and therefore an increase of the mass associated with the emission (by a factor of 6). However, the outer disk masses between two fixed radii (in the zone 10-50 AU) differ by less than a factor of 2. Thus, we conclude that uncertainties in the surface density slope introduce only a factor of a few uncertainty in our results.

We then consider the dependence of our results on different stellar UV luminosities. In addition to the UV flux for HD 35850 $\left(G_{0}=7.37 \times 10^{10}\right.$ at the stellar radius $\left.{ }^{9}\right)$ assumed previously, we have modeled two cases with 100 times higher and 100 times lower UV flux. The effect of higher stellar UV flux is to increase the heating (as well as alter the chemistry) and thus the predicted line luminosities of mid-infrared and millimeter lines (see Fig. 8). Note that an increase of 4 orders of magnitude in the UV flux increases the mid-infrared and millimeter line luminosities by factors smaller than 20 and 3, respectively. Because our method provides order-of-magnitude estimates of the stellar UV field, model line luminosities have only a factor of a few uncertainty. Based on the previous tests on the surface density slope, such luminosity uncertainties should have negligible effects on the limiting surface densities and on the extension of the emitting regions (compare Figs. 7 and 8 ).

Finally, we test the dependence of our results on the disk inner radius. Our earlier modeling of HD 105 (H05) showed that, for a range of $R_{\text {in }}$ between 1 and $40 \mathrm{AU}$, the upper limit on the surface density at the inner radius varied only by a factor of a few. This result is robust and can be extended to our sample: Spitzer data can set useful upper limits on the surface density for disks with $R_{\text {in }}$ out to 40 AU. ${ }^{10}$ For inner radii smaller than $1 \mathrm{AU}, \mathrm{H} 05$ found that the surface densities required to produce detectable lines

\footnotetext{
${ }^{9} G_{0}$ is the flux between 912 and $2000 \AA$ in units of $1.6 \times 10^{-3} \mathrm{ergs} \mathrm{cm}^{-2} \mathrm{~s}^{-1}$.

10 Note that $R_{\mathrm{em}}$ will change depending on $R_{\text {in }}$. The models from $\mathrm{H} 05$ suggest that the extension of the region where Spitzer lines originate is a few AU (see Table 2 of $\mathrm{H} 05$, where we used the notation $r_{w}$ instead of $R_{\mathrm{em}}$ ).
}

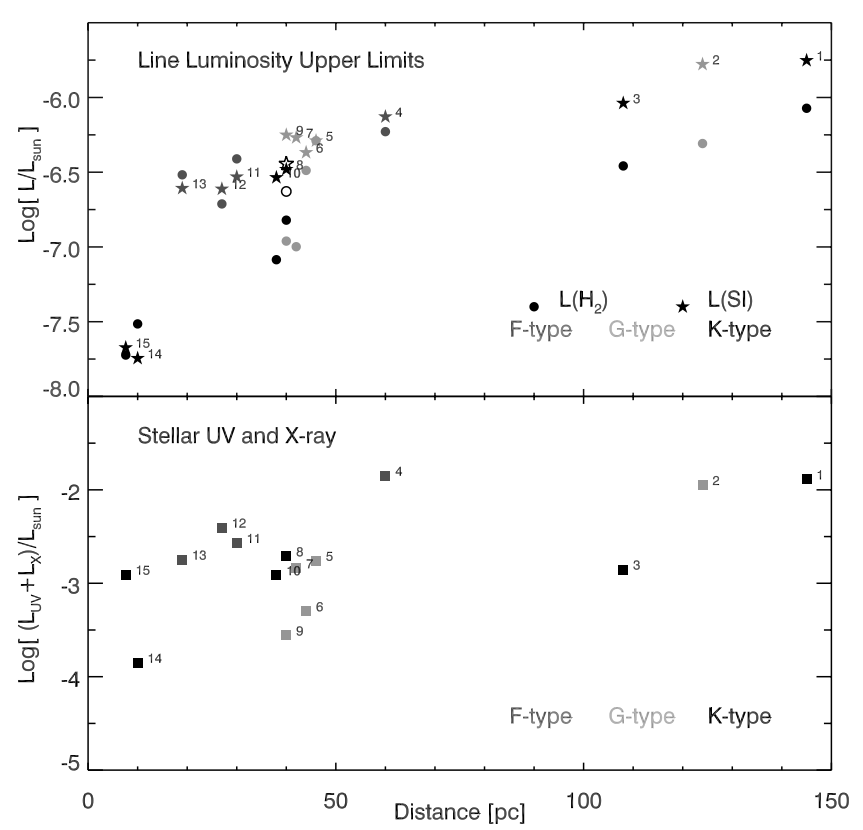

FIG. 9.- Top: Spitzer line luminosity upper limits vs. source distance. Stars and circles are for the $\left[\mathrm{S}_{\mathrm{I}}\right]$ and $\mathrm{H}_{2} S(1)$ transitions, respectively. For comparison we report with open symbols the upper limits to HD 105 (H05). Symbols in dark gray, light gray, and black indicate values from F-, G-, and K-type stars, respectively. Line luminosity limits are higher for the more distant sources. Bottom: Stellar UV and X-ray luminosities vs. source distance. Early-type stars and young/more distant sources have higher luminosities. [See the electronic edition of the Journal for a color version of this figure.]

increased (see their Fig. 6). This is because for smaller inner radii the mass or area (when lines are optically thick) of the emitting gas decreases. Essentially all of the heating is deposited close to the star and gas located at larger radii becomes too cool to produce any line emission at mid-infrared wavelengths. The result was that if the disk inner radius of HD 105 became smaller than $\sim 0.5$ AU, line upper limits from Spitzer could not set useful constraints on the gas mass.

In general, the sensitivity to the disk inner radius depends on the heating (stellar X-ray and UV) and on the line upper limits from Spitzer. While the younger sources in our sample typically have higher X-ray and UV luminosities and therefore produce more line flux, they are also more distant and so the upper limits on their luminosities are less stringent (see Fig. 9). To illustrate the effect of heating from stellar X-rays and UV, we use the two disks around the F-type star HD 35850 and the K-type star HD 17925. We have chosen these sources because they represent extremes in stellar UV and X-ray luminosity (HD 35850 has high UV and X-ray luminosities, whereas HD 17925 has low UV and X-ray luminosities) and have IUE spectra and CO millimeter data (sources 12 and 14 in Fig. 9). We consider five disk models with inner disk radii equal to $1,0.5,0.3,0.2$, and $0.1 \mathrm{AU}$ and compute for each case the surface density upper limits at the inner radius $\left(\Sigma_{0}\right)$ from the [S I] transition (Fig. 10, filled symbols $).{ }^{11}$ To show the effect of source distance, we also compute upper limits for HD 17925 at $30 \mathrm{pc}\left(\log \left[L\left(\mathrm{~S} \mathrm{I}_{\mathrm{I}}\right) / L_{\odot}\right]=-6.8\right)$ and $\mathrm{HD} 35850$ at $100 \mathrm{pc}\left(\log \left[L\left(\mathrm{~S} \mathrm{I}_{\mathrm{I}}\right) / L_{\odot}\right]=-5.5\right)$, i.e., at about 3 times their real distance (Fig. 10, open symbols). To calculate these line luminosities, we have assumed that the flux limits and the signal-to-noise ratio do not change with the source distance and simply scaled the luminosities with the distance square (in

\footnotetext{
${ }^{11}$ We have also computed the $R_{\mathrm{em}}$ from CO lines and noted only marginal changes with $R_{\text {in }}$.
} 


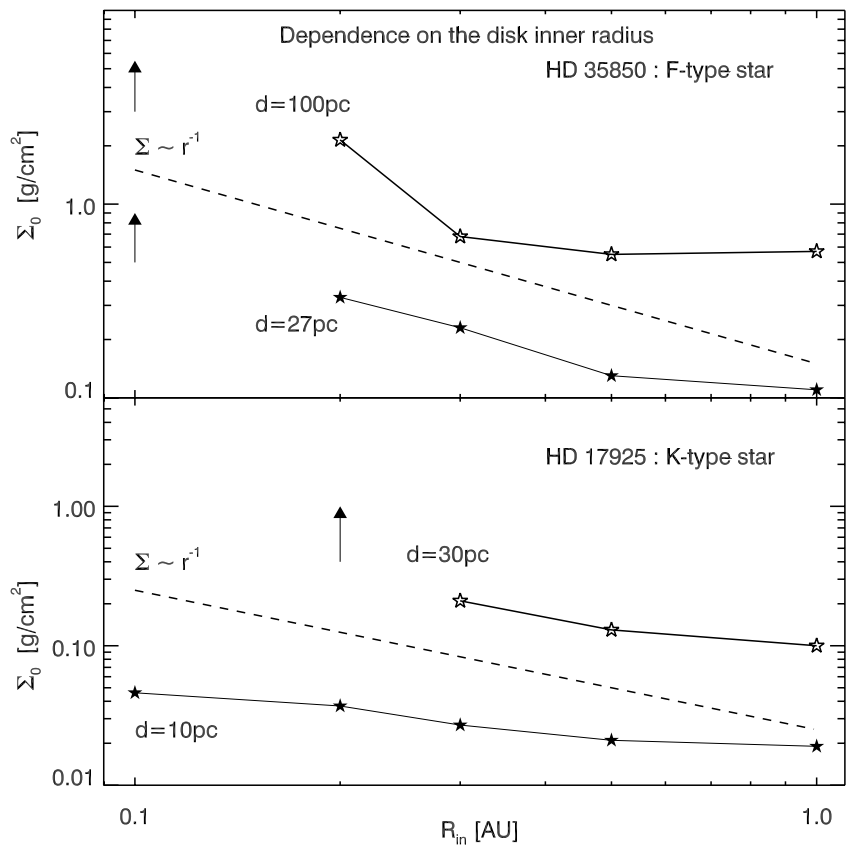

FIG. 10.-Dependence of the gas surface density upper limits $\left(\Sigma_{0}\right)$ on the disk inner radius. $\Sigma_{0}$ is calculated from the upper limits to the $\left[\mathrm{S}_{\mathrm{I}}\right]$ line at the source distance ( filled symbols) and at 30 and 100 pc for HD 17925 and HD 35850, respectively (open symbols). More distant sources have higher line luminosity upper limits that result in larger upper limits $\Sigma_{0}$ and in larger $R_{\text {in }}$ where the gas disk mass becomes unconstrained by Spitzer observations (this radius is marked by vertical arrows). For sources at a similar distance, upper limits to $\Sigma_{0}$ are set by the stellar X-ray and UV fields and more constraining limits are obtained for disks around early-type stars (compare the results for HD 35850 at $27 \mathrm{pc}$ and HD 17925 at $30 \mathrm{pc}$ ). The dashed line gives the dependence of our fiducial surface density with radius. The rise in $\Sigma_{0}$ for $R_{\text {in }}<0.5 \mathrm{AU}$ is steeper than the fiducial surface density slope for the more distant sources, implying somewhat higher surface density upper limits at $1 \mathrm{AU}$ for these disk inner radii.

reality our limits from the $\left[\mathrm{S}_{\mathrm{I}}\right]$ line are better for far away sources due to longer exposure times but only by a factor of 4 at most). These tests show that (1) more distant sources will typically have larger $\Sigma_{0}$ limits and larger disk inner radii at which the gas mass becomes unconstrained by Spitzer observations, and (2) high stellar heating lowers the limits on the gas surface density for sources at comparable distance (i.e., comparing HD 35850 at $27 \mathrm{pc}$ and HD 17925 at $30 \mathrm{pc}$, we see that we can set useful limits until $R_{\text {in }}=$ $0.2 \mathrm{AU}$ for the more luminous HD 35850). Given the Spitzer upper limits and the stellar properties of our sample (Fig. 9), we conclude that we can set useful upper limits to the gas mass in the majority of our systems for disks with inner holes ranging from $\sim 0.3$ to $40 \mathrm{AU}$. In the case of the more distant sources ScoPMS 214, MML 17, and MML 28, which are K- and G-type stars, the inner radius at which the surface density cannot be constrained may be larger than $0.3 \mathrm{AU}$ but still less than $1 \mathrm{AU}$.

\section{DISCUSSION}

We have shown that infrared and millimeter observations set low limits to the amount of gas in the inner $(\sim 1-5 \mathrm{AU})$ and outer ( $\geq 15 \mathrm{AU}$ ) regions, respectively, of our fiducial disks. In $\S 4.3$ we have proven that order-of-magnitude uncertainties in the stellar UV field and surface density slopes varying from -1.5 to -0.5 introduce only a factor of a few uncertainty in our results. Reducing the disk inner radius does not affect appreciably the result from the $\mathrm{CO}$ millimeter transitions because they originate farther out in the disk. On the other hand, mid-infrared lines are sensitive to small inner disk radii ( $<1 \mathrm{AU})$. Depending on the stellar properties, there is an inner radius at which Spitzer observations cannot set useful upper limits to the gas mass because the area or mass of the emitting gas is so small that lines cannot produce enough luminosity regardless of the surface density or the total disk mass. This critical inner radius is $\lesssim 0.3 \mathrm{AU}$ for the majority of our targets, and between 0.5 and $1 \mathrm{AU}$ for the three more distant systems (ScoPMS 214, MML 17, and MML 28). Thus, the Spitzer observations alone can exclude the existence of gas disks with inner holes $\gtrsim 0.3 \mathrm{AU}$ up to $\sim 40 \mathrm{AU}$ around the nearby $(d \leq 60 \mathrm{pc}) 12$ targets and gas disks with inner holes $\geq 1$ AU up to $\sim 40 \mathrm{AU}$ around the three targets at distances larger than $100 \mathrm{pc}$.

We want now to convert surface density upper limits from the [S I] line, the most sensitive mid-infrared transition, to gas mass upper limits and answer the question of whether the targeted disks have enough gas at their present age to form gas giant planets. For $R_{\text {in }} \gtrsim 1 \mathrm{AU}$, we have shown in the previous section that the $\left[\mathrm{S}_{\mathrm{I}}\right]$ line traces the inner few $\mathrm{AU}$ (with $3 \mathrm{AU}$ being the mean value from Table 8) and provides very similar surface density upper limits at the disk inner radius. Thus, we can use the $\Sigma_{0}$ at $1 \mathrm{AU}$ in Table 8 as representative of the limiting gas surface density at the inner radius for disks with $R_{\text {in }}$ between 1 and $40 \mathrm{AU}$. In this way, we find that Spitzer data can exclude gas masses larger than $\sim 0.04 M_{\mathrm{J}}$ within $3 \mathrm{AU}$ of the inner radius for disks with inner radii between 1 and $40 \mathrm{AU} .{ }^{12}$ This result is valid for all targets in our sample.

In addition, for the 12 nearby systems we can compute limits on the gas mass for disks with inner radii smaller than $1 \mathrm{AU}$. In the case of the nearby source HD 17925 (only $10 \mathrm{pc}$ away), which has very stringent line flux upper limits, we can exclude gas disks with inner holes as small as $0.1 \mathrm{AU}$ and a surface density at $1 \mathrm{AU}$ even smaller than those reported in Table 8 (see Fig. 10). This result can be extended to HD 216803, which is similarly nearby and experiences even higher heating (Fig. 9). More generally, limits on $\Sigma_{0}$ for $R_{\text {in }}<1 \mathrm{AU}$ increase faster than our assumed density slope and result in higher (up to a factor of 10 for $R_{\text {in }}$ close to $0.3 \mathrm{AU}$ ) surface density upper limits than those reported in Table 8 (see Fig. 10 and the HD 105 models from H05). However, even if assume that $\Sigma_{0}$ is 20 times larger at $0.3 \mathrm{AU}$ (which gives $5 \mathrm{~g} \mathrm{~cm}^{-2}$ ), we find that there is less than $0.0008 M_{\mathrm{J}}$ of gas between 0.3 and $1 \mathrm{AU}$; a constant surface density slope would have resulted in less than $0.002 M_{\mathrm{J}}$ of gas.

But could we also rule out a substantial amount $\left(\sim M_{\mathrm{J}}\right)$ of gas within $1 \mathrm{AU}$ in the case of the three more distant systems? A gaseous disk extending close to the star may be accreting. We can use the upper limits on the stellar accretion rates for these sources to constrain the amount of gas in this disk region. The mass accretion rate of a steady $\alpha$ disk around a solar-type star is $^{13}$

$$
\dot{M} \simeq 7 \times 10^{-9}\left(\frac{\alpha}{0.01}\right)\left(\frac{T_{1 \mathrm{AU}}}{100 \mathrm{~K}}\right)\left(\frac{\Sigma_{1 \mathrm{AU}}}{100 \mathrm{~g} \mathrm{~cm}^{-2}}\right)\left(M_{\odot} \mathrm{yr}^{-1}\right),
$$

where $\alpha$ is the viscosity parameter (typically 0.01 from magnetorotational instability models) and $T_{1 \mathrm{AU}}$ and $\Sigma_{1 \mathrm{AU}}$ are the gas disk temperature and the surface density at $1 \mathrm{AU}$, respectively. Accretion rates of $\sim 10^{-8} M_{\odot} \mathrm{yr}^{-1}$ are routinely measured

\footnotetext{
12 These masses are not very sensitive to the assumed gas surface density slopes because of the small (few $\mathrm{AU}$ ) region probed by mid-infrared gas lines.

${ }^{13}$ Eq. (2) was derived from eqs. (5.78), (5.64), and (5.31) of Hartmann (1998), assuming a gas surface density $\Sigma \propto r^{-1}$ and the isothermal sound speed for a hydrogen gas. We normalize the temperature to $100 \mathrm{~K}$ because that is close to the dust temperature in the midplane of accreting $\mathrm{T}$ Tauri disks.
} 
in disks surrounding young $(\sim 1 \mathrm{Myr})$ classical T Tauri stars by modeling the UV excess emission and the profile of the $\mathrm{H} \alpha$ emission lines. Muzerolle et al. (2000) have extended these techniques to 10 Myr old stars in the TW Hya association and detected accretion rates down to $\simeq 10^{-11} M_{\odot} \mathrm{yr}^{-1}$ for lower mass stars than are studied here. All of our targets have been observed in $\mathrm{H} \alpha$ with high spectral resolution and found to have only narrow $\mathrm{H} \alpha$ lines (equivalent widths $<5 \AA$ ) in absorption (White et al. 2006). The absence of $\mathrm{H} \alpha$ in emission indicates that they have already passed the phase of active gas accretion. We can use the $\mathrm{H} \alpha$ data for the three more distant sources and the minimum observable $\dot{M}$ from magnetospheric accretion models to infer an upper limit to the mass accretion rate. Magnetospheric accretion models predict that the minimum observable $\dot{M}$ depends on $R_{\star}^{(3 / 2)} M_{\star}^{(1 / 2)}$ and is about $10^{-10} M_{\odot} \mathrm{yr}^{-1}$ for a $1 \mathrm{Myr}$ old star with $M_{\star}=0.5 M_{\odot}$ and $R_{\star}=2 R_{\odot}(\mathrm{J}$. Muzerolle 2006, private communication). Given this dependence, the absence of $\mathrm{H} \alpha$ in emission, and the radii and masses of the stars, we can conclude that residual accretion may persist in these disks only at low rates $\lesssim 10^{-10} M_{\odot} \mathrm{yr}^{-1}$. Accretion rates $\lesssim 10^{-10} M_{\odot} \mathrm{yr}^{-1}$ translate into $\Sigma_{1 \mathrm{AU}} \lesssim 1.4 \mathrm{~g} \mathrm{~cm}^{-2}$ if the disk is accreting with $\alpha=0.01$ and $T_{1 \mathrm{AU}}$ is $100 \mathrm{~K}$ (eq. [2]). This surface density corresponds to a gas mass upper limit of $0.001 M_{\mathrm{J}}$ from the magnetospheric radius ( $3 R_{\odot}=0.01 \mathrm{AU}$ ) out to $1 \mathrm{AU}$ when adopting our fiducial disk surface density of $\Sigma \propto r^{-1}$. Although $\alpha=0.01$ and $T_{1 \mathrm{AU}}$ are extremely uncertain (and that is why upper limits from Spitzer data alone are valuable), it seems very unlikely that even the three more distant systems have large amounts of gas within $1 \mathrm{AU}$. Surface density limits at $0.3 \mathrm{AU}$ from the accretion are $\lesssim 5 \mathrm{~g} \mathrm{~cm}^{-2}$, comparable to Spitzer limits for nearby sources but much more stringent than what could be set from infrared data alone on these three more distant sources.

In summary, we can exclude gaseous reservoirs of $0.04 M_{\mathrm{J}}$ within a few AU of the disk inner radius for disk radii from 1 to $\sim 40$ AU. Spitzer data alone are sensitive to small amounts of gas from about 0.3 to $1 \mathrm{AU}$ for the majority of the sources. For the three more distant systems, accretion rate indicators set similarly stringent upper limits in the inner disk regions $(<1 \mathrm{AU})$. For disks with inner holes larger than $40 \mathrm{AU}$, mid-infrared transitions are not sensitive tracers of surface density because the gas is too cold. However, $\mathrm{CO}$ millimeter nondetections can exclude the presence of a large reservoir of cold gas at $\geq 20$ AU. In conclusion, there is no indication that the targeted disks have enough gas to form Jupiter- or Saturn-like planet(s). The present data cannot exclude the possibility that some or all of these systems have already formed such planets.

What can we learn about the gas dissipation timescale based on the above discussion? Our sample includes sources that are beyond the phase of active gas accretion. In addition, their small infrared excesses suggest that they have already dissipated most of their primordial dust and/or agglomerated it into larger particles. Our results show that by this time most of the gas has also been dispersed perhaps by photoevaporation (e.g., Clarke et al. 2001) and/or accreted to form gas giant planets. These conclusions are consistent with a rapid gas dissipation timescale that leaves little trace of gas in optically thin dust disks. More statistics on sources younger than $10 \mathrm{Myr}$ in age and also on sources with optically thick dust disks are necessary to observationally constrain the gas lifetime and explore any link between gas and dust dissipation.

Short gas dissipation timescales are relevant not only to the formation of giant planets but also to the later stages of terrestrial planet formation. These final stages of growth may have involved tens to hundreds of planetary embryos over a timescale of

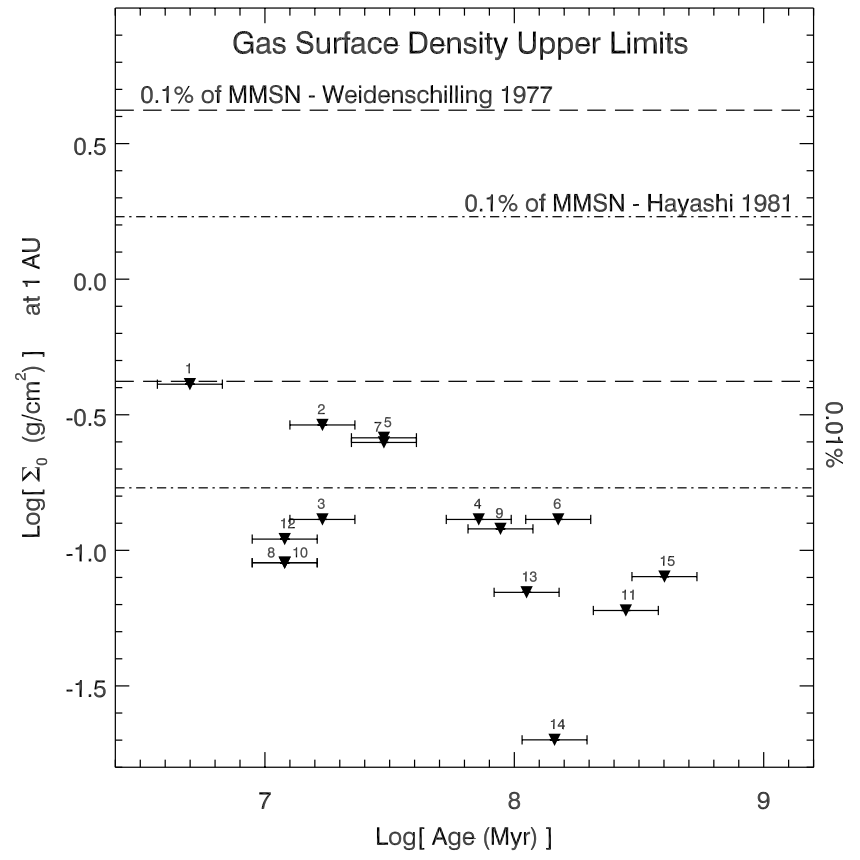

FIG. 11.- Gas surface density upper limits vs. age compared to the surface density of the MMSN model from Weidenschilling (1977; dashed lines) and Hayashi (1981; dot-dashed lines). Surface density upper limits are from the [S I] line for a disk with $R_{\text {in }}=1 \mathrm{AU}$ (see Table 8); ages and error bars are from Table 1 . Our surface density upper limits at 1 AU are lower than $0.01 \%$ of the MMSN value from Weidenschilling (1977) for all sources. Note that the anticorrelation between the density upper limits at the inner radius and age does not depend on our model assumptions but rather on the properties of our sample. Because young sources are more distant than old ones, our line luminosity upper limits are less stringent for young disks (see Table 1 and Fig. 9).

10-100 Myr (Canup 2004). Numerical simulations find that terrestrial planets grow to Earth size in timescales of tens of million of years, in agreement with isotopic constraints (Kleine et al. 2002). A problem in the simulations of these later stages is that secular perturbations by (the presumably already formed) Jupiter and Saturn and by neighboring embryos excite terrestrial planet eccentricities. ${ }^{14}$ Thus, some damping mechanisms seem to be required to circularize the final orbits of terrestrial planets. One possibility is through tidal interactions with a remnant gas disk. Kominami \& Ida (2002) suggest that small amounts of gas, between $0.1 \%$ and $0.01 \%$ of the minimum mass solar nebula (MMSN; Weidenschilling 1977; Hayashi 1981), can reduce the eccentricities to values as low as those of Earth and Venus on a timescale of about $10 \mathrm{Myr}$ if secular perturbations from Jupiter and Saturn do not have a significant effect on the evolution (Kominami \& Ida 2004). Alternatively, dynamical friction associated with remnant swarms of planetesimals can damp the eccentricities over longer timescales even in the presence of perturbations from the giant planets (O’Brien et al. 2006; Raymond et al. 2006). Thus, our low gas surface density upper limits at 1 AU (see Fig. 11) suggest that circularization of terrestrial planets if it occurs beyond $10 \mathrm{Myr}$ may have happened primarily through the latter mechanism, although certainly gas could have played a role during the earlier stages of terrestrial planet formation.

Finally, the gas limits in the $10-40 \mathrm{AU}$ region, the region mainly traced by our millimeter $\mathrm{CO}$ observations, may also be

\footnotetext{
14 Collisions with large impactors (up to the mass of Mars) occur frequently at average times of $\sim 30 \mathrm{Myr}$ and random orientations but seem to cause smaller changes in the eccentricities than secular perturbations (see, e.g., Chambers 2001).
} 
relevant to the formation of outer gas-poor giant planets such as Uranus and Neptune. Because of the dynamical interactions between embryos and the gravitational effects of Jupiter and Saturn, very little accretion of solids is expected to have occurred at the location of Uranus and Neptune in the absence of mechanisms to increase capture cross sections of embryos (Levison \& Stewart 2001). One way to do this is through gas drag. However, this mechanism would require many Jupiter masses of gas (e.g., Goldreich et al. 2004) that are not observed in any of our targets (see Tables 8 and 9). The $\mathrm{CO}$ data for eight of our sources (with ages between 12 and few hundred Myr) indicate that less than 2 $M_{\oplus}$ of gas are present between 10 and $40 \mathrm{AU}$ in our disks. These values are smaller than the gas mass of Uranus and Neptune as inferred by models of their interiors (Guillot 1999). Thus, if Uranus and Neptune formed at their current radii, either gas persisted much longer in the solar nebula than was the case for our target stars, or they formed relatively quickly, in much less than 100 Myr.

\section{SUMMARY AND CONCLUSIONS}

We analyzed infrared and millimeter spectra for 15 of our FEPS sources selected for investigating the gas dispersal timescale in disks around solar-type stars. Our targets span a wide age range from 5 to a few hundred Myr (with 50\% of the sample younger than $\sim 30 \mathrm{Myr}$ ), and most are surrounded by optically thin dust disks. We did not detect gas lines in the Spitzer IRS modules or in the millimeter with the SMT. We estimated upper limits to the gas mass using simple approximations and also more sophisticated gas and dust models. In agreement with our previous modeling of HD 105 (H05), we find that optically thin dust disks have too little dust surface density to appreciably heat the gas. Therefore, X-ray flux and UV flux from the central star become the main heating mechanisms for the gas. We show that gas line upper limits from Spitzer provide sensitive limits to the gas surface density at the disk inner radius. Millimeter CO data are complementary by setting limits on the gas mass in the outer disk ( $\geq 15$ AU). We have also discussed the robustness of our results by varying the main uncertain input parameters to the disk models. Future work will include a study weighted toward disks younger than $10 \mathrm{Myr}$ and tests of the gas models on sources with detected gas lines probing different disk regions. Our main conclusions can be summarized as follows:

1. Simple estimates of gas mass upper limits from mid-infrared $\mathrm{H}_{2}$ lines indicate that even the five youngest disks (5-20 Myr) in our sample have less than $0.6 M_{\mathrm{J}}$ of gas at $100 \mathrm{~K}$. Detailed gas models of the infrared and millimeter upper limits combined with the absence of accretion signatures allow us to conclude that none of the targeted disks have enough gas to form Jupiter- or Saturnlike planets at the present time.

2. Our results do not support the presence of "large quantities of gas in debris disks" as proposed by Thi et al. (2001b). On the contrary, we have evidence that systems with small dust excess in the 10-30 Myr age range do not have large amounts of remnant gas.

3. We estimate gas surface density upper limits at $1 \mathrm{AU}$ smaller than $0.01 \%$ of the MMSN model for most of the sources, eight of which have ages between 5 and 30 Myr. If the circularization of terrestrial planets occurs in this age range, then gas surface densities appear to be so low that interactions with planetesimals seem to be the only viable mechanism to circularize their orbits.

4. The gas limits from $\mathrm{CO}$ data in the 10-40 AU region are less than a few $M_{\oplus}$. These values are far too low for gas drag to enhance the gravitational cross section of embryos and speed up the in situ formation of Uranus and Neptune. In addition, if these systems are analogs of the solar system, our results indicate relatively short timescales ( $\ll 100 \mathrm{Myr})$ for the formation of Uranusand Neptune-like planets.

It is a pleasure to thank all members of the FEPS team for their contributions to the project and to this study. I. P. wishes to thank D. Watson for suggestions on the data reduction of the IRS highresolution spectra and J. Muzerolle for helpful discussions on the mass accretion rate in young circumstellar disks. We would also like to thank the anonymous referee for a very careful and helpful review. This work is based on observations made with the Spitzer Space Telescope, which is operated by the Jet Propulsion Laboratory, California Institute of Technology under NASA contract 1407. Support for this work was provided by NASA through the FEPS Legacy award issued by JPL/Caltech.

\section{Facilities: Spitzer}

\section{REFERENCES}

Apai, D., Pascucci, I., Bouwman, J., Natta, A., Henning, T., \& Dullemond, C. P. 2005, Science, 310, 834

Bary, J. S., Weintraub, D. A., \& Kastner, J. H. 2003, ApJ, 586, 1136

Bouwman, J., Meeus, G., de Koter, A., Hony, S., Dominik, C., \& Waters, L. B. F. M. 2001, A\&A, 375, 950

Bryden, G., et al. 2006, ApJ, 636, 1098

Canup, R. M. 2004, ARA\&A, 42, 441

Chambers, J. E. 2001, Icarus, 152, 205

Chen, C. H. 2006, in ASP Conf. Ser. 352, New Horizons in Astronomy: Frank N. Bash Symposium 2005, ed. S. J. Kannappan et al. (San Francisco: ASP), 63 Chen, C. H., et al. 2006, ApJS, in press (astro-ph/0605277)

Chiang, E. I., \& Goldreich, P. 1997, ApJ, 490, 368

Clarke, C. J., Gendrin, A., \& Sotomayor, M. 2001, MNRAS, 328, 485

de Zeeuw, P. T., Hoogerwerf, R., de Bruijne, J. H. J., Brown, A. G. A., \& Blaauw, A. 1999, AJ, 117, 354

Dent, W. R. F., Greaves, J. S., \& Coulson, I. M. 2005, MNRAS, 359, 663

Draine, B. T., \& Lee, H. M. 1984, ApJ, 285, 89

Dutrey, A., \& Guilloteau, S. 2004, Ap\&SS, 292, 407

Dutrey, A., Guilloteau, S., Duvert, G., Prato, L., Simon, M., Schuster, K., \& Menard, F. 1996, A\&A, 309, 493

Dutrey, A., Lecavelier Des Etangs, A., \& Augereau, J.-C. 2004, in Comets II, ed. M. C. Festou, H. U. Keller, \& H. A. Weaver (Tucson: Univ. Arizona Press), 81

Fajardo-Acosta, S. B., Stencel, R. E., Backman, D. E., \& Thakur, N. 1999, ApJ, 520,215
Gautier, D., Hersant, F., Mousis, O., \& Lunine, J. I. 2001, ApJ, 550, L227

Goldreich, P., Lithwick, Y., \& Sari, R. 2004, ARA\&A, 42, 549

Gorti, U., \& Hollenbach, D. 2004, ApJ, 613, 424 (GH04)

Gray, R. O., Corbally, C. J., Garrison, R. F., McFadden, M. T., Bubar, E. J., McGahee, C. E., O'Donoghue, A. A., \& Knox, E. R. 2006, AJ, 132, 161

Gray, R. O., Corbally, C. J., Garrison, R. F., McFadden, M. T., \& Robinson, P. E. 2003, AJ, 126, 2048

Guillot, T. 1999, Science, 286, 72

Habing, H. J. 1969, Bull. Astron. Inst. Netherlands, 20, 177

Habing, H. J., et al. 2001, A\&A, 365, 545

Hartmann, L. 1998, Accretion Processes in Star Formation (Cambridge: Cambridge Univ. Press)

Hayashi, C. 1981, Prog. Theor. Phys. Suppl., 70, 35

Herczeg, G. J., Linsky, J. L., Valenti, J. A., Johns-Krull, C. M., \& Wood, B. E. 2002, ApJ, 572, 310

Higdon, S. J. U., et al. 2004, PASP, 116, 975

Hollenbach, D., et al. 2005, ApJ, 631, 1180 (H05)

Houk, N. 1982, Michigan Catalogue of Two-dimensional Spectral Types for the HD Stars, Vol. 3 (Ann Arbor: Dept. Astronomy, Univ. Michigan)

Houk, N., \& Cowley, A. P. 1975, Michigan Catalogue of Two-dimensional Spectral Types for the HD Stars (Ann Arbor: Dept. Astronomy, Univ. Michigan)

Houk, N., \& Smith-Moore, M. 1988, Michigan Catalogue of Two-dimensional Spectral Types for the HD Stars, Vol. 4 (Ann Arbor: Dept. Astronomy, Univ. Michigan) 
Houk, N., \& Swift, C. 1999, Michigan Catalogue of Two-dimensional Spectral Types for the HD Stars, Vol. 5 (Ann Arbor: Dept. Astronomy, Univ. Michigan) Huensch, M., Schmitt, J. H. M. M., \& Voges, W. 1998, A\&AS, 132, 155

Jacobsen, S. B. 2005, Annu. Rev. Earth Planet. Sci., 33, 531

Jaschek, M. 1978, Bull. Cent. Donnees Stellaires, 15, 121

Jonkheid, B., Faas, F. G. A., van Zadelhoff, G.-J., \& van Dishoeck, E. F. 2004, A\&A, 428, 511

Keller, L. P., et al. 2002, Nature, 417, 148

Kim, J. S., et al. 2005, ApJ, 632, 659

Kleine, T., Münker, C., Mezger, K., \& Palme, H. 2002, Nature, 418, 952

Kominami, J., \& Ida, S. 2002, Icarus, 157, 43 2004, Icarus, 167, 231

Lecavelier des Etangs, A., et al. 2001, Nature, 412, 706

Levison, H. F., \& Stewart, G. R. 2001, Icarus, 153, 224

Makarov, V. V. 2003, AJ, 126, 1996

Mamajek, E. E., Meyer, M. R., \& Liebert, J. 2002, AJ, 124, 1670

Mathis, J. S. 1990, ARA\&A, 28, 37

Meyer, M. R., Backman, D. E., Weinberger, A. J., \& Wyatt, M. C. 2006, in Protostars and Planets V, in press (astro-ph/0606399)

Micela, G., Favata, F., \& Sciortino, S. 1997, A\&A, 326, 221

Moór, A., Ábrahám, P., Derekas, A., Kiss, C., Kiss, L. L., Apai, D., Grady, C., \& Henning, T. 2006, ApJ, 644, 525

Muzerolle, J., Calvet, N., Briceño, C., Hartmann, L., \& Hillenbrand, L. 2000, ApJ, 535, L47

Najita, J., Carr, J. S., \& Mathieu, R. D. 2003, ApJ, 589, 931

Najita, J., \& Williams, J. P. 2005, ApJ, 635, 625

O'Brien, D. P., Morbidelli, A., \& Levison, H. F. 2006, Icarus, 184, 39

Pascucci, I., Wolf, S., Steinacker, J., Dullemond, C. P., Henning, T., Niccolini, G., Woitke, P., \& Lopez, B. 2004, A\&A, 417, 793

Perryman, M. A. C., et al. 1997, A\&A, 323, L49

Pollack, J. B., Hollenbach, D., Beckwith, S., Simonelli, D. P., Roush, T., \& Fong, W. 1994, ApJ, 421, 615

Preibisch, T., Brown, A. G. A., Bridges, T., Guenther, E., \& Zinnecker, H. 2002, AJ, 124, 404

Przygodda, F., van Boekel, R., Àbrahàm, P., Melnikov, S. Y., Waters, L. B. F. M., \& Leinert, C. 2003, A\&A, 412, L43

Raymond, S. N., Quinn, T., \& Lunine, J. I. 2006, Icarus, 183, 265

Ribas, I., Guinan, E. F., Güdel, M., \& Audard, M. 2005, ApJ, 622, 680

Richter, M. J., Jaffe, D. T., Blake, G. A., \& Lacy, J. H. 2002, ApJ, 572, L161
Rieke, G. H., et al. 2005, ApJ, 620, 1010

Roberge, A., Weinberger, A. J., Redfield, S., \& Feldman, P. D. 2005, ApJ, 626, L105

Sako, S., Yamashita, T., Kataza, H., Miyata, T., Okamoto, Y. K., Honda, M., Fujiyoshi, T., \& Onaka, T. 2005, ApJ, 620, 347

Savage, B. D., \& Sembach, K. R. 1996, ARA\&A, 34, 279

Schmitt, J. H. M. M., \& Liefke, C. 2004, A\&A, 417, 651

Scoville, N. Z., Sargent, A. I., Sanders, D. B., Claussen, M. J., Masson, C. R., Lo, K. Y., \& Phillips, T. G. 1986, ApJ, 303, 416

Sheret, I., Ramsay Howat, S. K., \& Dent, W. R. F. 2003, MNRAS, 343, L65

Shu, F. H., Adams, F. C., \& Lizano, S. 1987, ARA\&A, 25, 23

Song, I., Zuckerman, B., \& Bessell, M. S. 2003, ApJ, 599, 342

Spangler, C., Sargent, A. I., Silverstone, M. D., Becklin, E. E., \& Zuckerman, B. 2001, ApJ, 555, 932

Sternberg, A., \& Neufeld, D. A. 1999, ApJ, 516, 371

Su, K. Y. L., et al. 2005, ApJ, 628, 487

Tagliaferri, G., Cutispoto, G., Pallavicini, R., Randich, S., \& Pasquini, L. 1994, A\&A, 285, 272

Takeuchi, T., \& Artymowicz, P. 2001, ApJ, 557, 990

Thi, W. F., et al. 2001a, ApJ, 561, 1074 2001b, Nature, 409, 60

Valenti, J. A., Fallon, A. A., \& Johns-Krull, C. M. 2003, ApJS, 147, 305

Valenti, J. A., Johns-Krull, C. M., \& Linsky, J. L. 2000, ApJS, 129, 399

Voges, W., et al. 1999, A\&A, 349, 389

Walter, F. M., Vrba, F. J., Mathieu, R. D., Brown, A., \& Myers, P. C. 1994, AJ, 107,692

Walter, F. M., et al. 2003, AJ, 126, 3076

Weidenschilling, S. J. 1977, Ap\&SS, 51, 153

White, R. J., Gabor, J., \& Hillenbrand, L. A. 2006, AJ, in press

Wichmann, R., Schmitt, J. H. M. M., \& Hubrig, S. 2003, A\&A, 399, 983

Wood, B. E., Redfield, S., Linsky, J. L., Müller, H.-R., \& Zank, G. P. 2005, ApJS, 159, 118

Wyatt, M. C., Dermott, S. F., Telesco, C. M., Fisher, R. S., Grogan, K., Holmes, E. K., \& Piña, R. K. 1999, ApJ, 527, 918

Zuckerman, B., Forveille, T., \& Kastner, J. H. 1995, Nature, 373, 494

Zuckerman, B., \& Song, I. 2004, ARA\&A, 42, 685

Zuckerman, B., Song, I., Bessell, M. S., \& Webb, R. A. 2001, ApJ, 562, L87

Zuckerman, B., \& Webb, R. A. 2000, ApJ, 535, 959 